\title{
(Q,S,s) PRICING RULES
}

Kenneth Burdett

Guido Menzio

Working Paper 19094

http://www.nber.org/papers/w19094

\section{NATIONAL BUREAU OF ECONOMIC RESEARCH 1050 Massachusetts Avenue Cambridge, MA 02138}

May 2013

We are grateful to Roland Benabou, Ben Eden, Allen Head, Greg Kaplan, Moritz Meyer-ter-Vehn, Pierre-Olivier Weill and, especially, Randy Wright for useful discussions on early versions of the paper. We are also grateful to seminar participants at Princeton University, UCLA, UCL, the Econometric Society Meeting (Evanston 2012) and the Search and Matching Workshop (Philadelphia 2012). The views expressed herein are those of the authors and do not necessarily reflect the views of the National Bureau of Economic Research.

NBER working papers are circulated for discussion and comment purposes. They have not been peerreviewed or been subject to the review by the NBER Board of Directors that accompanies official NBER publications.

(C) 2013 by Kenneth Burdett and Guido Menzio. All rights reserved. Short sections of text, not to exceed two paragraphs, may be quoted without explicit permission provided that full credit, including (C) notice, is given to the source. 
(Q,S,s) Pricing Rules

Kenneth Burdett and Guido Menzio

NBER Working Paper No. 19094

May 2013

JEL No. D11,D21,D43,E32

\begin{abstract}
$\underline{\text { ABSTRACT }}$
We study the effect of menu costs on the pricing behavior of sellers and on the cross-sectional distribution of prices in the search-theoretic model of imperfect competition of Burdett and Judd (1983). We find that, when menu costs are small, the equilibrium is such that sellers follow a $(\mathrm{Q}, \mathrm{S}, \mathrm{s})$ pricing rule. According to a $(\mathrm{Q}, \mathrm{S}, \mathrm{s})$ rule, a seller lets inflation erode the real value of its nominal price until it reaches some point $\mathrm{s}$. Then, the seller pays the menu cost and changes its nominal price so that the real value of the new price is randomly drawn from a distribution with support $[S, Q]$, where $Q$ is the buyer's reservation price and $\mathrm{S}$ is some price between $\mathrm{s}$ and $\mathrm{Q}$. Only when the menu cost is relatively large, the equilibrium is such that sellers follow a standard $(\mathrm{S}, \mathrm{s})$ pricing rule. We argue that whether sellers follow a $(\mathrm{Q}, \mathrm{S}, \mathrm{s})$ or an $(\mathrm{S}, \mathrm{s})$ rule matters for the estimation of menu costs and seller-specific shocks.
\end{abstract}

Kenneth Burdett

University of Pennsylvania

kennethb@econ.upenn.edu

Guido Menzio

Department of Economics

University of Pennsylvania

467 McNeil Building

3718 Locust Walk

Philadelphia, PA 19104

and NBER

gmenzio@econ.upenn.edu 


\section{Introduction}

In menu cost models, the optimal pricing strategy of a seller is almost always given by an $(S, s)$ rule. That is, a seller lets inflation erode the real value of its nominal price until it reaches some point $s$. Then the seller pays the menu cost and changes its nominal price so that the real value of the new price is some $S$ greater than $s$. The optimality of an $(S, s)$ pricing rule has been established in a wide variety of environments (see, e.g., Sheshinski and Weiss 1977, Caplin and Spulber 1987, Dotsey, King and Wolman 1999). Indeed, the optimality of an $(S, s)$ rule seems to be such a natural outcome that some authors simply assume it (see, e.g, Caplin and Leahy 1991). After all, what else could sellers possibly do?

In this study, we introduce menu costs in a simple search-theoretic model of imperfect competition in the spirit of Butters (1977), Varian (1980) and Burdett and Judd (1983). We show that, as long as the menu cost is sufficiently low, the optimal pricing strategy of a seller is not an $(S, s)$ rule. The optimal pricing strategy is what we call a $(Q, S, s)$ rule. According to a $(Q, S, s)$ rule, a seller lets inflation erode the real value of its nominal price until it reaches some point $s$. Then, the seller pays the menu cost and changes its nominal price so that the real value of the new price is a random draw from a distribution with some support $[S, Q]$, where $Q$ is strictly greater than $S$ and $S$ is strictly greater than $s$.

In the model analyzed in this study, sellers post nominal prices whose real value is eroded by inflation and that can only be changed by paying a menu cost. Buyers search for sellers. In some meetings buyers are captive, in the sense that they are in contact with only one seller, while in other meetings they are not, in the sense that they are in contact with multiple sellers. Without menu costs, it is well known that the equilibrium of this model generates price dispersion (see, e.g, Burdett and Judd 1983). With a small menu cost, we find that the equilibrium is unique and such that sellers follow a $(Q, S, s)$ pricing rule. We refer to this as a $(Q, S, s)$ equilibrium. With a large menu cost, we find that all equilibria are such that seller follow an $(S, s)$ rule, where the seller's highest price $S$ is equal to the buyer's reservation price $Q$. We refer to this as an $(S, s)$ equilibrium. With intermediate values of the menu cost, we find that $(Q, S, s)$ and $(S, s)$ equilibria may coexist. Finally, we argue that the economic forces that rule out an $(S, s)$ equilibrium when menu costs are small are exactly the same forces that rule out a unique price equilibrium when there are no menu costs. 
A $(Q, S, s)$ equilibrium has several distinctive features. First, the seller's present discounted value of profits remains constant as the real value of the nominal price falls from $Q$ to $S$, and declines monotonically as the real value of the nominal price falls from $S$ to $s$. Second, the seller's flow profit remains constant as the real value of its nominal price falls from $Q$ to $S$, then increases, peaks and declines. Third, the stationary distribution of prices is a combination of the distribution that obtains in standard menu cost models and the distribution that obtains in static search-theoretic models of price dispersion. In particular, over the interval $[s, S]$, the stationary distribution of prices is log-uniform, as in the standard menu costs models (see, e.g., Caplin and Spulber 1987 or Benabou 1988). Over the interval $[S, Q]$, the stationary distribution of prices is such that the seller's flow profit is constant, as in the static search models of price dispersion (see, e.g., Butters 1977, Varian 1980 or Burdett and Judd 1983). Fourth, the mixing distribution from which sellers draw their new prices is the only one that generates a stationary price distribution that makes sellers indifferent between resetting their price anywhere between $S$ and $Q$.

The notion of a $(Q, S, s)$ pricing rule is not just an intellectual curiosity. In a $(Q, S, s)$ equilibrium, a seller maintains the same nominal price until its real value reaches some point $s$. While the real value of the nominal price falls from $Q$ to $S$, the seller would not want to change the price even if it could do it for free. This follows as any real price between $Q$ and $S$ yields the same discounted value of profits. Only while the real value of the nominal price falls from $S$ to $s$, would the seller like to change its price but chooses not because of the menu cost. In this sense, only part of the price stickiness generated by the model is caused by the menu cost. Indeed, even as the menu cost goes to zero, the model generates sticky prices. In contrast, in an $(S, s)$ equilibrium, all of the price stickiness generated by the model is due to menu costs. Therefore, if an econometrician were to incorrectly assume an $(S, s)$ rather than a $(Q, S, s)$ equilibrium, he would estimate incorrectly large menu costs from the observed duration of prices.

Moreover, in a $(Q, S, s)$ equilibrium, a seller changes its nominal price by different amounts at different times, even though it always faces the same demand and always produces with the same technology. Sometimes, the seller may adjusts its nominal price by as little as $(S-s) / s$ percent. At other times, the seller may adjusts its nominal price by as much as $(Q-s) / s$ percent. In contrast, in an $(S, s)$ equilibrium, the seller always changes its nominal price by the same amount. Therefore, if an econometrician were to incorrectly assume an $(S, s)$ rather than a $(Q, S, s)$ equilibrium, he would estimate incorrectly large shocks to an individual seller's demand or technology from the observed variation in the 
magnitude of nominal price adjustments. These observations are particularly relevant because, as it turns out, the real effect of monetary shocks tends to be larger precisely when menu costs and seller-specific shocks are larger (see, e.g., Golosov and Lucas 2007, Klenow and Kryvstov 2008 or Midrigan 2011).

Our paper contributes to three strands of literature. First, our paper contributes to the literature on menu costs. Sheshinski and Weiss (1977) consider the problem of a monopolist facing a well-behaved downward sloping demand curve. They show that the seller follows an $(S, s)$ rule and characterize the bounds $S$ and $s$. Caplin and Spulber (1987) and Caplin and Leahy $(1997,2010)$ consider models in which a continuum of monopolists faces an exogenously given demand curve which depends on their own real price, on the aggregate price and on the supply of money. They prove that each monopolist follows an $(S, s)$ rule and that the cross-sectional distribution of prices is log-uniform. Dotsey, King and Wolman (1999) and Golosov and Lucas (2007) and Midrigan (2011) consider models in which a continuum of monopolistic competitors faces an endogenous demand curve derived from the buyers' Dixit-Stiglitz preferences. They show that each monopolistic competitor follows an $(S, s)$ rule. Finally, Benabou $(1988,1992)$ introduces menu costs in the search model of Diamond (1971). He shows that the optimal pricing strategy of the sellers is an $(S, s)$ rule, where $S$ is given by the buyers' reservation price $Q$ and $s$ is determined by the magnitude of the menu cost. All of the papers listed above consider environments where, absent menu costs, all identical sellers would set the same price. Our paper is the first to consider an environment where, absent menu costs, identical sellers would set different prices. This is the reason why we find that the optimal pricing strategy of a seller may be a $(Q, S, s)$ rule rather than an $(S, s)$ rule.

Second, our paper contributes to the literature on search-theoretic models of price dispersion. Butters (1977) considers an environment in which some buyers contact only one seller and some contact two sellers. Since they cannot discriminate between the two types of buyers, sellers find it optimal to randomize with respect to their price. Varian (1980) considers an environment in which some buyers contact one seller and some buyers contact all the sellers. Again, sellers find it optimal to randomize with respect to their price. Burdett and Judd (1983) consider an environment in which buyers choose how many sellers to contact. They show that there exists an equilibrium in which some buyers choose to contact one seller, some buyers choose to contact two sellers and sellers randomize over their price. In all of the papers mentioned above, the seller's problem is static because 
sellers are free to change their price in every period. In our paper, the seller's problem is dynamic because of menu costs.

Third, our paper contributes to the literature on the real effect of monetary shocks in menu cost models of price stickiness. Caplin and Spulber (1987) argue that money supply shocks have no effect on the real side of the economy when the cross-sectional price distribution is log-uniform. Dotsey, King and Wolman (1999) and Golosov and Lucas (2007) and Midrigan (2011) argue that positive shocks to the money supply have favorable effects on the real side of the economy when the cross-sectional price distribution has a lower density around s than a log-uniform distribution. Moreover, they show that the density of the cross-sectional price distribution around s is smaller when sellers face larger idiosyncratic shocks and when menu costs are higher. Our paper shows that, if sellers follow a $(Q, S, s)$ rule rather than an $(S, s)$ rule, the typical estimates of idiosyncratic shocks and menu costs are likely to be biased upwards.

Head, Liu, Menzio and Wright (2012) consider a monetary version of Burdett and Judd (1983) without menu costs. They show that the equilibrium uniquely pins down the distribution of real prices, but not the pricing strategy of individual sellers. In particular, as the aggregate money supply increases, not every seller needs to change its price even though all sellers are free to do so. In fact, if the real value of its nominal price remains on the support of the equilibrium distribution, the seller is indifferent between changing and not changing price. Only if the real value of its nominal price is pushed out of the support of the equilibrium distribution, the seller must change price. They show that, for some specifications of the seller's pricing behavior, the model is consistent with all the empirical behavior of individual prices documented by Klenow and Kryvstov (2008) and Steinsson and Nakamura (2008). They also show that, in the model, monetary shocks are perfectly neutralized by changes in the equilibrium price distribution. Therefore, they argue that it is not correct to conclude that there must be menu costs because individual prices are sticky. Similarly, they argue that it is not correct to conclude that monetary shocks must have real effects because individual prices are sticky. Head et al. (2012) assume that there are no menu costs and, for this reason, the equilibrium is largely indeterminate. In our paper, we assume that menu costs exist, although they may be arbitrarily small and, for this reason, the equilibrium pins down both the price distribution and the seller's individual behavior.

The remainder of the paper is organized as follows. Section 2 describes the environment 
and compares it with Burdett and Judd (1983) and Benabou (1988). Section 3 derives a necessary and sufficient condition for the existence of a $(\mathrm{Q}, \mathrm{S}, \mathrm{s})$ equilibrium, characterizes the salient features of the equilibrium and carries out comparative statics with respect to the menu cost and the inflation rate. Section 4 derives a necessary and sufficient condition for the existence of an $(\mathrm{S}, \mathrm{s})$ equilibrium and it shows that this type of equilibrium does not exist when the menu cost is sufficiently small. Section 5 briefly concludes.

\section{Environment}

We study a dynamic and monetary version of a model of imperfect competition in the spirit of Butters (1977), Varian (1980) and Burdett and Judd (1983). The market for an indivisible good is populated by a continuum of identical sellers with measure 1. Each seller maximizes the present value of real profits, discounted at the rate $r>0$. Each seller produces the good at a constant marginal cost, which, for the sake of simplicity, we assume to be zero. Each seller posts a nominal price d for the good, which can only be changed by paying the real cost $c$, with $c>0$.

The market is also populated by a continuum of identical buyers. In particular, during each interval of time of length $d t$, a measure $b d t$ of buyers enters the market. A buyer comes into contact with one seller with probability $\alpha$ and with two sellers with probability $1-\alpha$, where $\alpha \in(0,1)$. We refer to a buyer who contacts only one seller as captive, and to a buyer who contacts two sellers as non-captive. Then, the buyer observes the nominal prices posted by the contacted sellers and decides whether and where to purchase a unit of the good. If the buyer purchases the good at the nominal price $d$, he obtains a utility of $Q-\mu(t) d$, where $\mu(t)$ is the utility value of a dollar at date $\mathrm{t}$ and $Q>0$ is the buyer's valuation of the good. If the buyer does not purchase the good, he obtains a reservation utility, which we normalize to zero. Whether the buyer purchases the good or not, he exits the market.

The utility value of a dollar declines at the constant rate $\pi>0$. Therefore, if a nominal price remains unchanged during an interval of time of length $d t$, the real value of the price falls by $\exp (-\pi d t)$. In this paper, we do not describe the demand and supply of dollars. It would, however, be straightforward to embed our model into either a standard cashin-advance framework (see, e.g., Lucas and Stokey 1987) or in a standard money-search 
framework (see, e.g., Lagos and Wright 2005) and show that, in a stationary equilibrium, the depreciation rate pie would be equal to the growth rate of the money supply.

Even without inflation and menu costs, the equilibrium of the model features a nondegenerate distribution of prices. The logic behind this result is clear. If all sellers post the same price, an individual seller can increase its profits by charging a slightly lower price and sell not only to the contacted buyers who are captive, but also to the contacted buyers who are not captive. This Bertrand-like process of undercutting cannot push all prices down to the marginal cost. In fact, if all sellers post a price equal to the marginal cost, an individual seller can increase its profits by charging the reservation price $Q$ and sell only to the contacted buyers who are captive. Thus, in equilibrium, there must be price dispersion.

There are two differences between our model and Burdett and Judd (1983) (henceforth, BJ83). First, in our model sellers post nominal prices that can only be changed by paying a menu cost, while in BJ83 sellers post real prices that can be freely changed in every period. This difference is important because it implies that in our model the problem of the seller is dynamic, while in BJ83 it is static. Second, in our model the fraction of buyers meeting one and two sellers is exogenous, while in BJ83 it is an endogenous outcome of buyers' optimization. This should not be a substantive difference, in that we believe that our results would go through even if buyers' search intensity was endogenous. There are also two differences between our model and Benabou (1988) (henceforth, B88). First, in our model there are some buyers who are in contact with one seller and some who are in contact with multiple sellers, while in B88 all buyers are temporarily captive. This difference is important because it implies that, even without menu costs, the equilibrium of our model features price dispersion, while in B88 every seller would charge the monopoly price (as in Diamond 1971). Second, in our model buyers have to leave the market after their first search, while in B88 they can choose to stay in the market and search again.

This should not be a substantive difference because our results should go through even if buyers were allowed to search repeatedly.

\section{$3(Q, S, s)$ Equilibrium}

A $(Q, S, s)$ equilibrium is an equilibrium where every seller lets inflation erode the real value of its price until it reaches some point $s \in(0, Q)$, then it pays the menu cost and it 
resets the nominal price so that its real value is a random draw from some distribution with support $[S, Q]$, where $S \in(s, Q)$. In a $(Q, S, s)$ equilibrium, the stationary distribution of real prices is some continuous distribution $F$ with support $[s, Q]$ and the distribution of new prices is some distribution $G$ with support $[S, Q]$. In subsection 3.1 , we formally define a $(Q, S, s)$ equilibrium. In subsection 3.2 , we derive a necessary and sufficient condition for the existence of a $(Q, S, s)$ equilibrium. In subsection 3.3, we describe several novel features of a $(Q, S, s)$ equilibrium, as well as their implications for the estimation of menu costs, seller-specific shocks and search costs. In subsection 3.4, we analyze the effect on equilibrium outcomes of changes in the menu cost and in the inflation rate. The main result of this section is that, as long as menu costs are not too large, a $(Q, S, s)$ equilibrium exists and is unique. ${ }^{1}$

\section{1 $(Q, S, s)$ Equilibrium: Definition}

Consider a seller whose nominal price has a real value of $Q \exp (-\pi t)$. Let $V(t)$ denote the present discounted value of the real profits of this seller, which is given by

$$
V(t)=\max _{T}\left\{\int_{t}^{T} e^{-r(x-t)} R\left(Q e^{-\pi x}\right) d x+e^{-r(T-t)}\left(V^{*}-c\right)\right\},
$$

where

$$
R\left(Q e^{-\pi x}\right)=b\left[\alpha+2(1-\alpha)\left(1-F\left(Q e^{-\pi x}\right)\right)\right] Q e^{-\pi x}
$$

As time goes from 0 to $T-t$, the real value of the seller's nominal price falls at the rate $\pi$. After $x-t$ units of time, the real price of the seller is $Q \exp (-\pi x)$ and its real profit is $R(Q \exp (-\pi x))$. The real profit of the seller is the sum of two terms. The first term is given by the arrival rate of a buyer who is not in contact with any other seller, $b \alpha$, times the probability that the buyer is willing to purchase from the seller, 1, times the seller's profit in case of a sale, $Q \exp (-\pi x)$. The second term is given by the arrival rate of a seller who is in contact with another firm, $2 b(1-\alpha)$, times the probability that the buyer is willing to purchase from the seller, $1-F(Q \exp (-\pi x))$, times the seller's profit in case of a sale, $Q \exp (-\pi x)$. After $T-t$ units of time, the seller pays the menu cost $c$, resets the nominal price and attains the maximized value $V^{*}$.

\footnotetext{
${ }^{1}$ We do not need to look for equilibria in which a seller resets the real value of its nominal price to an interval $[S, P]$, where $P<Q$. Indeed, it is easy to verify that this class of equilibria does not exist because, if $F(P)$ were equal to 1 , an individual seller would be strictly better off resetting the real value of its nominal price to $Q$ rather than to $P$. Similarly, we can rule out equilibria in which a seller randomizes over the real value $p$ of the nominal price at which it pays the menu cost. All details are available upon request.
} 
The seller finds it optimal to change the nominal price after $T^{*}-t$ units of time, where $T^{*}$ is such that

$$
R\left(Q e^{-\pi T^{*}}\right)=r\left(V^{*}-c\right)
$$

The left-hand side of (3) is the marginal benefit of deferring the price change, which is given by the expected profit associated with the real price $Q \exp (-\pi x)$. The right-hand side of (3) is the marginal cost of deferring the price change, which is given by the annuitized value of paying the menu cost $c$ and attaining the maximum continuation value $V^{*}$. Thus, $T^{*}$ is such that the marginal benefit and the marginal cost of deferring the price change are equated. From (3), it follows that the seller finds it optimal to change the nominal price when its real value reaches $s$ if and only if $s=Q \exp \left(-\pi T^{*}\right)$ or, equivalently,

$$
R(s)=r\left(V^{*}-c\right)
$$

The seller finds it optimal to reset the nominal price to any real value between $S$ and $Q$ if and only if $V(t)=V^{*}$ for all $t \in\left[0, T_{1}\right]$, and $V(t) \leq V^{*}$ for all $t \in\left[T_{1}, T_{1}+T_{2}\right]$, where $T_{1}=\log (Q / S) / \pi$ denotes the time it takes for the real value of a nominal price to travel from $Q$ to $S$ and $T_{2}=\log (S / s) / \pi$ denotes the time it takes for the real value of a nominal price to travel from $S$ to $s$. The condition $V(t)=V^{*}$ for all $t \in\left[0, T_{1}\right]$ is equivalent to

$$
\begin{gathered}
\int_{T_{1}}^{T_{1}+T_{2}} e^{-r\left(x-T_{1}\right)} R\left(Q e^{-\gamma x}\right) d x+e^{-r T_{2}}\left(V^{*}-c\right)=V^{*} \\
R\left(Q e^{-\pi t}\right)=r V^{*}, \forall t \in\left[0, T_{1}\right] .
\end{gathered}
$$

Equation (5) states that the present discounted value of profits for a seller with a real price of $S=Q \exp \left(-\pi T_{1}\right)$ is equal to the maximum value $V^{*}$. Equation (6) states that the profit of the seller when its real price is $Q \exp (-\pi t)$ is equal to the annuitized value of $V^{*}$ for all $t \in\left[0, T_{1}\right]$. Since $r V(t)=R(Q \exp (-\pi t))+V^{\prime}(t)$ and $V\left(T_{1}\right)=V^{*}$, this implies that $V^{\prime}(t)=0$ for all $t \in\left[0, T_{1}\right]$. Taken together equations (5) and (6) are equivalent to the condition $V(t)=V^{*}$ for all $t \in\left[0, T_{1}\right]$. Similarly, the condition $V(t) \leq V^{*}$ for all $t \in\left[T_{1}, T_{1}+T_{2}\right]$ is equivalent to

$$
\int_{t}^{T_{1}+T_{2}} e^{-r(x-t)} R\left(Q e^{-\pi x}\right) d x+e^{-r\left(T_{1}+T_{2}-t\right)}\left(V^{*}-c\right) \leq V^{*}
$$

The distribution of prices, $F$, is stationary if the measure of sellers whose real price 
enters the interval $[s, p]$ is equal to the measure of sellers whose real price exits the interval $[s, p]$ during any arbitrarily small period of time $d t$. For any $p \in(s, S)$, the inflow-outflow equation is given by

$$
F\left(e^{\gamma d t} p\right)-F(p)=F\left(e^{\gamma d t} s\right)-F(s)
$$

The flow of sellers into the interval $[s, p]$ is equal to the measure of sellers who, at the beginning of the period, have a real price between $p$ and $p \exp (\pi d t)$. Each one of these sellers sees the real value of its price fall below $p$ during a period of time of length $d t$. The flow of sellers out of the interval $[s, p]$ is equal to the measure of sellers who, at the beginning of the period, have a real price between $s$ and $s \exp (\pi d t)$. Each one of these sellers pays the menu cost and resets the nominal price so that its real value is somewhere in the interval $[S, Q], S>p$. Dividing both sides of (8) by $d t$ and taking the limit $d t \rightarrow 0$, we obtain

$$
F^{\prime}(p) p=F^{\prime}(s) s, \forall p \in(s, S)
$$

For any $p \in(S, Q)$, the inflow-outflow equation is given by

$$
F\left(e^{\gamma d t} p\right)-F(p)=\left[F\left(e^{\gamma d t} s\right)-F(s)\right][1-G(p)]
$$

The flow of sellers into the interval $[s, p]$ is equal to the measure of sellers who, at the beginning of the period, have a real price between $p$ and $p \exp (\pi d t)$. Each one of these sellers sees the real value of its price fall below $p$ during a period of time of length $d t$. The flow of sellers out of the interval $[s, p]$ is equal to a fraction $1-G(p)$ of the measure of sellers who, at the beginning of the period, have a real price between $s$ and $s \exp (\pi d t)$. Every seller with a real price between $s$ and $s \exp (\pi d t)$ pays the menu cost and resets its nominal price. A fraction $1-G(p)$ of them resets the nominal price so that its real value is greater than $p$. Dividing both sides of (10) by $d t$ and taking the limit $d t \rightarrow 0$, we obtain

$$
F^{\prime}(p) p=F^{\prime}(s) s[1-G(p)], \forall p \in(S, Q)
$$

The stationary price distribution $F$ must satisfy the differential equation (9) over the interval $(s, S)$ and the differential equation (11) over the interval $(S, Q)$. In addition, the stationary price distribution $F$ must satisfy the boundary conditions

$$
F(s)=0 \text { and } F(Q)=1 .
$$

The above observations motivate the following definition of equilibrium. 
Definition 1. A stationary $(Q, S, s)$ equilibrium is a $C D F$ of prices $F:[s, Q] \rightarrow[0,1], a$ $C D F$ of new prices $G:[S, Q] \rightarrow[0,1]$, a lower bound on the price distribution $s \in(0, Q)$, a lower bound on the new price distribution $S \in(s, Q)$, and a seller's maximum value $V^{*}$ that jointly satisfy the optimality conditions (4)-(7) and the stationarity conditions (9), (11) and (12).

\section{2 $(Q, S, s)$ Equilibrium: Existence}

The equilibrium condition (6) implies that $R(Q)$, the seller's profit when the real value of its nominal price is $Q$, must be equal to $r V^{*}$, the annuitized maximum value of the seller. Since $R(Q)$ is given by $b[\alpha+2(1-\alpha)(1-F(Q))] Q$ and $F(Q)$ is equal to 1 , the maximum value of the seller is equal to

$$
V^{*}=\frac{b \alpha Q}{r} .
$$

The seller's maximum value is equal to the present discounted value of profits for a hypothetical seller that always charges the buyer's reservation price $Q$ and never has to incur the menu cost. The fact that the seller's maximum value is independent of the menu cost is a surprising property of the equilibrium. Intuitively, the property obtains because any increase in the menu cost is entirely passed through to the buyers through the response of the equilibrium distribution of prices.

The equilibrium condition (6) states that $R(p)$ must be equal to $r V^{*}$ for all $p \in[S, Q]$. As $R(p)$ is given by $b[\alpha+2(1-\alpha)(1-F(p))] p$ and $r V^{*}$ is equal to $b \alpha Q$, it follows that the stationary price distribution is equal to

$$
F(p)=1-\frac{\alpha}{2(1-\alpha)} \frac{Q-p}{p}, \forall p \in[S, Q] .
$$

Over the interval $[S, Q]$, the stationary price distribution is exactly the same as in BJ83. This property of the equilibrium follows from the fact that, as in BJ 83, the stationary price distribution is such that the seller's profits are constant over the interval $[S, Q]$.

The equilibrium condition (9) states that the derivative of the price distribution $F^{\prime}(p)$ must be equal to $F^{\prime}(s) s / p$ for all $p \in(s, S)$. Integrating this equilibrium condition on $F^{\prime}(p)$ and using the fact that $F(s)$ is equal to 0 , we find that the stationary price distribution is equal to

$$
F(p)=\left[1-\frac{\alpha}{2(1-\alpha)} \frac{Q-S}{S}\right] \frac{\log (p / s)}{\log (S / s)}, \forall p \in[s, S]
$$


where the second line makes use of the continuity condition $F(S-)=F(S+)$. Over the interval $[s, S]$, the stationary price distribution is log-uniform, exactly as in B88 and in other models where sellers follow an $(S, s)$ pricing rule. This property of the equilibrium follows from the fact that, as in B88, sellers enter the interval $[s, S]$ from the top, they travel through the interval $[s, S]$ at the constant rate $\pi$, and they exit the interval $[s, S]$ from the bottom. As first argued by Caplin and Spulber (1987), the log-uniform distribution is the only stationary distribution consistent with this type of behavior.

The equilibrium condition (11) states that the derivative of the price distribution $F^{\prime}(p)$ must be equal to $F^{\prime}(s) s(1-G(p)) / p$ for all $p \in(S, Q)$. As we know that $F^{\prime}(p)$ is equal to the derivative of the right-hand side of (14), we can solve the equilibrium condition (11) with respect to the distribution of new prices $G$ and obtain

$$
G(p)=1-\left(1-\frac{\alpha}{2(1-\alpha)} \frac{Q-S}{S}\right)^{-1} \frac{\alpha \log (S / s) Q}{2(1-\alpha) p}, \forall p \in(S, Q) .
$$

The role of the distribution of new prices $G$ is to generate the cross-sectional distribution of prices $F$ that makes the seller's profit and value constant over the interval $[S, Q]$ and, hence, makes sellers indifferent between resetting their price anywhere in the interval $[S, Q]$. Interestingly, in order to fulfill its role, the distribution of new prices must have mass points at $S$ and $Q$ with measure

$$
\begin{gathered}
\chi(S)=1-\left(1-\frac{\alpha}{2(1-\alpha)} \frac{Q-S}{S}\right)^{-1} \frac{\alpha \log (S / s) Q}{2(1-\alpha) S}, \\
\chi(Q)=\left(1-\frac{\alpha}{2(1-\alpha)} \frac{Q-S}{S}\right)^{-1} \frac{\alpha \log (S / s)}{2(1-\alpha)} .
\end{gathered}
$$

Notice that, although the distribution of new prices has two mass points, the stationary price distribution is everywhere continuous. Intuitively, $F$ is continuous because the fraction of sellers who reset their price to $S$ and $Q$ is strictly positive, but the measure of sellers who reset their price in a particular instant is zero.

Finally, we need to solve for the equilibrium prices $s$ and $S$. The equilibrium condition (4) states that $R(s)$, the profit associated with the lowest price $s$, must be equal to $r\left(V^{*}-c\right)$, the annuitized value of paying the menu cost and resetting the nominal price. Since $R(s)$ is equal to $b[\alpha+2(1-\alpha)] s$ and $r\left(V^{*}-c\right)$ is equal to $b \alpha Q-r c$, the lowest price $s$ is equal to

$$
s=\frac{\alpha Q-r c / b}{2-\alpha}
$$


The equilibrium condition (6) states that the present discounted value of profits for a seller with a real price $S$ must equal the maximum value $V^{*}$. After substituting out $F$, $G$ and $V^{*}$ and after solving the integral, we can rewrite the equilibrium condition (6) as

$$
\begin{aligned}
\varphi(S) \equiv & {\left[\frac{1-e^{-(r+\pi) T_{2}(S)}\left(1+(r+\pi) T_{2}(S)\right)}{(r+\pi)^{2}}\right] \frac{(2-\alpha) S-\alpha Q}{T_{2}(S)} } \\
& +\left[\frac{1-e^{-(r+\pi) T_{2}(S)}}{r+\pi}\right] \alpha Q+e^{-r T_{2}(S)}\left(\frac{\alpha Q}{r}-\frac{c}{b}\right)-\frac{\alpha Q}{r}=0 .
\end{aligned}
$$

where

$$
T_{2}(S) \equiv \frac{\log (S / s)}{\pi}, \quad s=\frac{\alpha Q-r c / b}{2-\alpha}
$$

A necessary condition for the existence of a $(Q, S, s)$ equilibrium is that the equation $\varphi(S)=0$ admits a solution for some $S$ in the interval $(s, Q)$. Since $\varphi(S)$ is strictly negative for $S \in[s, \alpha Q /(2-\alpha)]$ and strictly increasing for $S \in[\alpha Q /(2-\alpha), Q]$, a necessary condition for the existence of a $(Q, S, s)$ equilibrium is that $\varphi(Q)$ is strictly positive. As stated in Theorem 1, this condition is also sufficient.

Theorem 1: (Existence of a $(Q, S, s)$ equilibrium). $A(Q, S, s)$ equilibrium exists iff $\varphi(Q)>0$. Moreover, if a $(Q, S, s)$ equilibrium exists, it is unique and: (a) $V^{\prime}(t)=0$ for all $t \in\left(0, T_{1}\right)$ and $V^{\prime}(t)<0$ for all $t \in\left(T_{1}, T_{1}+T_{2}\right) ;(b) \hat{R}^{\prime}(t)=0$ for all $t \in\left(0, T_{1}\right), \hat{R}^{\prime}(t)>0$ for all $t \in\left(T_{1}, \hat{T}\right)$ and $\hat{R}^{\prime}(t)<0$ for all $t \in\left(T, T_{1}+T_{2}\right)$, where $\hat{R}(t) \equiv R(Q \exp (-\pi t))$ and $\hat{T} \in\left(T_{1}, T_{1}+T_{2}\right)$.

Proof: In Appendix A.

First, it is useful to note that the condition $\varphi(Q)>0$ - which is necessary and sufficient for the existence of a $(Q, S, s)$ equilibrium - does not define an empty set of parameter values. For instance, it is straightforward to verify that the condition is satisfied when the inflation rate $\pi$ is sufficiently low or when the menu cost $c$ is sufficiently small.

Second, it is useful to sketch the proof of the sufficiency of condition $\varphi(Q)>0$. If $\varphi(Q)>0$, the equation $\varphi(S)=0$ admits a solution $S^{*} \in(\alpha Q /(2-\alpha), Q)$. Let $s^{*}$ be given as in equation (19) for $S=S^{*}$. Notice that $s^{*}$ is strictly positive as $c<b \alpha Q / r$ and that $s^{*}$ is strictly less than $S^{*}$ as $s^{*}<\alpha Q /(2-\alpha)$. Let $F^{*}$ be given as in equations (14) and (15) for $S=S^{*}$ and $s=s^{*}$. Notice that $F^{*}$ is a proper cumulative distribution function with support $\left[s^{*}, Q\right]$ because $F\left(s^{*}\right)=0, F^{*}(Q)=1$ and $F^{* \prime}(p)>0$ for all $p \in\left[s^{*}, Q\right]$. Let $G^{*}$ be given as in equations (16)-(18) for $S=S^{*}$ and $s=s^{*}$. It is easy to verify 


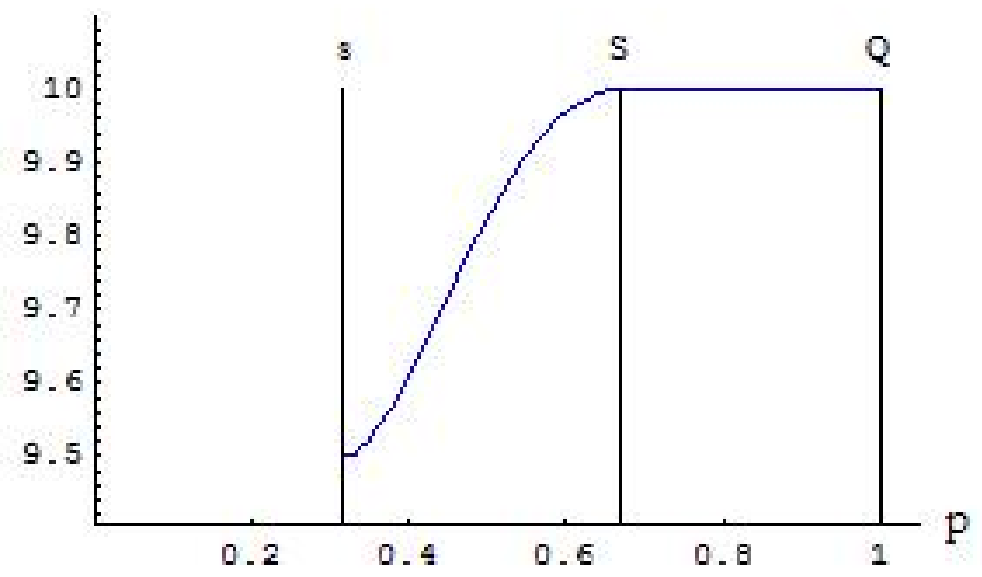

Figure 1: Present value of profits

that $G^{*}$ is a proper cumulative distribution function with support $\left[s^{*}, S^{*}\right]$. Finally, let $V^{*}$ be given as in (13). Because of the way equations (13)-(20) have been constructed, the tuple $\left(F^{*}, G^{*}, S^{*}, s^{*}, V^{*}\right)$ satisfies the optimality conditions (4)-(6) and the stationarity conditions (9), (11) and (12).

It remains to be seen that the tuple $\left(F^{*}, G^{*}, S^{*}, s^{*}, V^{*}\right)$ satisfies the optimality condition (7), i.e. the present discounted value of profits for a seller with a real price between $s$ and $S$ is non-greater than $V^{*}$. To this aim, we first notice that the equilibrium price distribution $F^{*}$ guarantees that, as the real price falls from $S^{*}$ to $s^{*}$, the seller's flow profit $R(p)$ increases, peaks and then decreases. Second, we prove that this property together with the fact that $V^{*}=b \alpha Q / r$ implies that, as the real price falls from $S^{*}$ to $s^{*}$, the seller's present discounted value of profits declines.

\subsection{Properties and Implications of a $(Q, S, s)$ Equilibrium}

Figures 1 through 4 illustrate the main qualitative features of a $(Q, S, s)$ equilibrium. Figure 1 plots the seller's value as a function of the real price. As the real value of the nominal price falls from $Q$ to $S$, the seller's value remains constant and equal to the maximum $V^{*}$. As the real value of the nominal price falls from $S$ to $s$, the seller's value monotonically declines from $V^{*}$ to $V^{*}-c$. Hence, when the real price reaches $s$, the seller finds it optimal to pay the menu cost. 


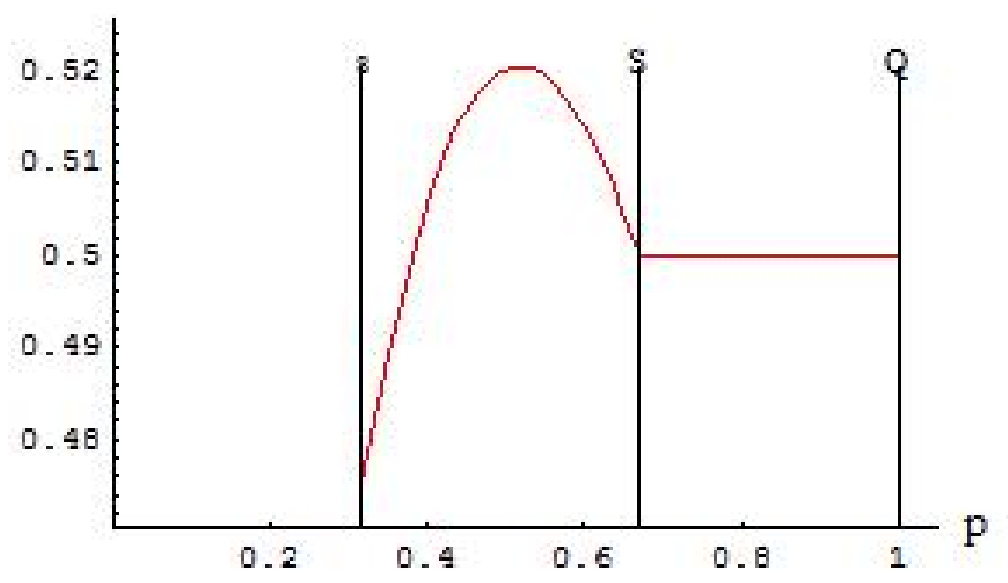

Figure 2: Flow profit

When the seller pays the menu cost, it is indifferent between resetting its nominal price to any real value between $S$ and $Q$. At first blush, this property of the equilibrium may seem puzzling as the seller would have to pay the menu cost less frequently if it were to reset the real price to $Q$ rather than to, say, $S$. The solution to the puzzle is contained in Figure 2, which plots the seller's flow profit as a function of the real price. As the real price falls from $Q$ to $S$, the seller's flow profit is constant and equal to $r V^{*}$, which guarantees that the derivative of the seller's value function is zero. As the real price falls below $S$, the seller's flow profit begins to increase, it reaches a maximum, it begins to fall and, eventually, it attains the value $r\left(V^{*}-c\right)$. Thus, if the seller resets its real price to $Q$ rather than to some lower value, it will pay the menu cost less frequently but it will also enjoy the highest flow profit less frequently. The two effects exactly balance each other and, for this reason, the seller is indifferent between resetting its real price to any value between $S$ and $Q$.

Figure 3 plots the stationary distribution of prices. Over the interval $[s, S]$, the distribution is log-uniform as in standard $(S, s)$ models. Over the interval $[S, Q]$, the distribution is such that the seller's flow profit is constant as in standard search models of equilibrium price dispersion. At the border between the two intervals (i.e. at the price $S$ ), there is a kink in the distribution and, consequently, a discontinuity in the density. In particular, the density of prices to the right of $S$ is discontinuously lower than the density of prices to the left of $S$. This discontinuity is the reason why the seller's flow profit increases when the real price falls below $S$. In general, when the real price falls, the seller experiences an 


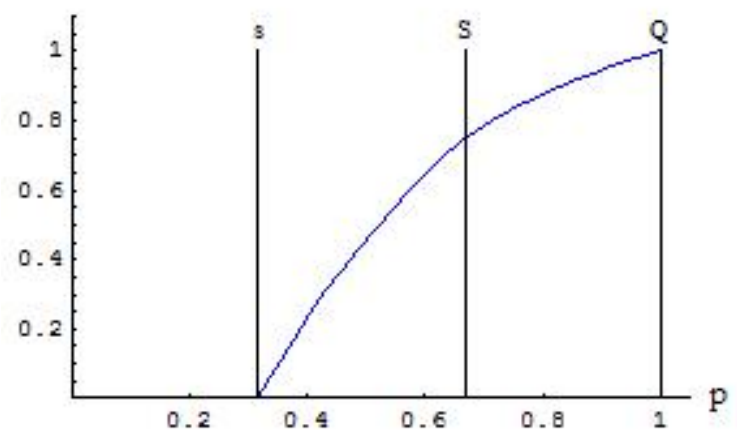

(a) $\mathrm{CDF}$

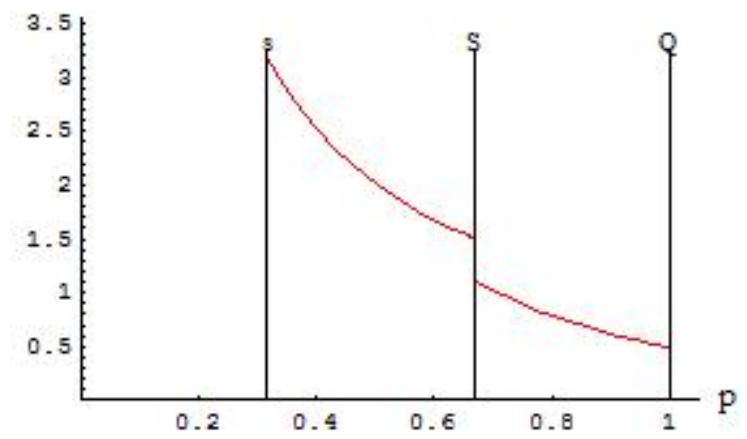

(b) PDF

Figure 3: Price distribution F

increase in volume that is proportional to the increase in the fraction of firms charging a price higher than the seller's. That is, when its real price falls, the seller experiences an increase in volume proportional to the density of the price distribution. As the real price falls from $Q$ to $S$, the density of the price distribution is such that the increase in the seller's volume exactly offsets the decline in the seller's price. As the real price falls below $S$, the density of the price distribution jumps up and, hence, the increase in the seller's volume more than offsets the decline in the seller's price and the seller's flow profit increases.

Figure 4 plots the distribution of new prices. The support of the distribution of new prices is the interval $[S, Q]$. The distribution has a mass point at $S$, another mass point at $Q$, and it is continuous everywhere else. The fact that the distribution of new prices has a mass point at $S$ is the reason why the stationary price distribution has a kink at $S$, why the density of the stationary price distribution has a discontinuity at $S$ and, in turn, why the seller's flow profit increases when its real price falls below $S$. Similarly, the fact that the distribution of new prices has a mass point at $Q$ is the reason why the density of the stationary price distribution is strictly positive at $S$, which is a necessary condition for the seller's flow profit to be non-decraesing when the real price falls below $Q$.

A $(Q, S, s)$ equilibrium has also have important consequences for the estimation of menu cost models (see, e.g., Golosov and Lucas 2007, Klenow and Kryvtsov 2008 or Midrigan 2011). Let us briefly illustrate this point. In a $(Q, S, s)$ equilibrium, every time a seller chooses a new nominal price, it picks a real value $p$ from the distribution $G$. The seller's new nominal price lasts for $\log (p / s) / \pi$ units of time. During the first $\log (p / S) / \pi$ 


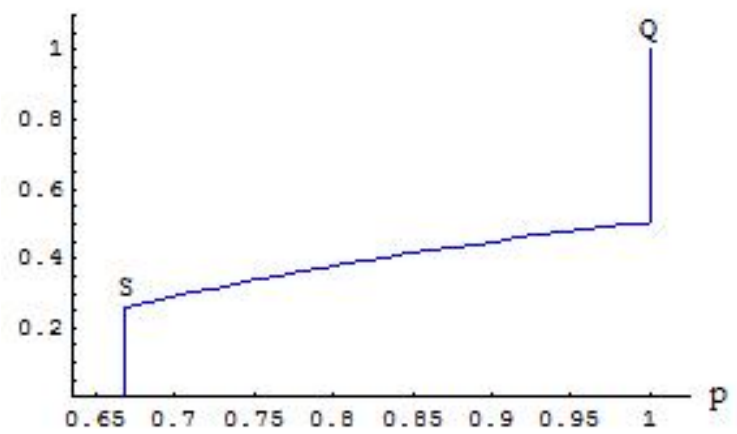

(a) $\mathrm{CDF}$

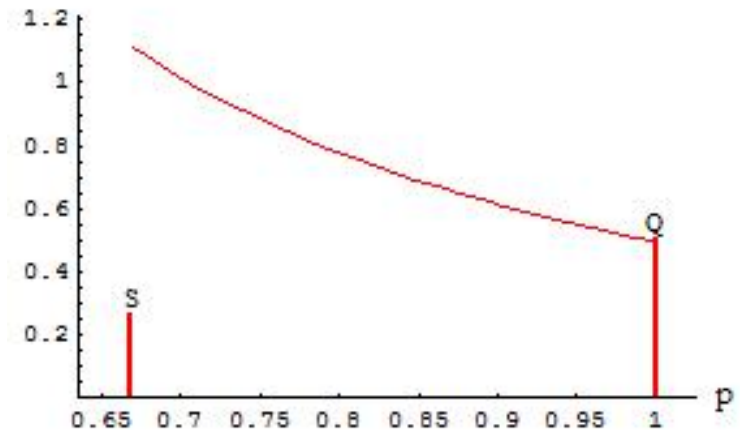

(b) PDF

Figure 4: New price distribution G

units of time, the seller would not want to change its nominal price even if it could do it for free. During the last $\log (S / s) / \pi$ units of time, the seller would like to change its nominal price, but it chooses not to because of the menu cost. In this sense, only part of the "stickiness" of the seller's nominal price is caused by menu costs. This property of the equilibrium suggests that, if an econometrician were to incorrectly assume that sellers follow an $(S, s)$ pricing rule rather than a $(Q, S, s)$ pricing rule, he would estimate incorrectly large menu costs from the empirical duration of prices. Clearly, this estimation bias could be very severe if $\log (Q / S)$ was very large relative to $\log (S / s)$.

In a $(Q, S, s)$ equilibrium, when a seller chooses a new nominal price, the magnitude of the price change is given by $\log (p / s)$, where $p$ is drawn from the distribution $G$. That is, in a $(Q, S, s)$ equilibrium, a seller changes its nominal price by a different percentage at different times even though it always faces the same demand and always produces with the same technology. This property of equilibrium implies that, if an econometrician were to incorrectly assume that sellers follow an $(S, s)$ pricing rule rather than a $(Q, S, s)$ pricing rule, he would estimate incorrectly large seller-specific productivity shocks from the empirical variance of price changes across sellers.

Overall, if an econometrician were to incorrectly assume that sellers follow an $(S, s)$ rule, he would likely overestimate both the magnitude of menu costs and the magnitude of seller-specific productivity shocks. In turn, these two estimation biases may lead to overestimate the importance of sticky prices in explaining the response of the real economy to monetary shocks. In fact, as it transpires from the analysis in Dotsey, King and Wolman (1997), Golosov and Lucas (2007), Klenow and Kryvstov (2008) and Midrigan (2011), in 
models of state-dependent pricing, monetary shocks tend to generate larger real effects precisely when menu costs and idiosyncratic shocks are larger. ${ }^{2}$

The notion of a $(Q, S, s)$ equilibrium may also have an impact on the estimation of search costs (see, e.g., Hong and Shum 2006 and Moraga-Gonzales and Wildenbeest 2008). The distribution of search costs among buyers is typically estimated using the empirical price distribution and the identifying assumption that sellers who post different prices must expect an identical profit. This identifying assumption is consistent with equilibrium if sellers can adjust their price for free, but it is not if sellers face menu costs. Indeed, in a $(Q, S, s)$ equilibrium, the seller's profit is constant only over the interval $[S, Q]$. Therefore, if an econometrician were to incorrectly abstract from menu costs, he would obtained biased estimates of the distribution of search costs among buyers.

\section{4 $(Q, S, s)$ Equilibrium: Comparative Statics}

In the previous subsections, we have established a necessary and sufficient condition for the existence of a $(Q, S, s)$ equilibrium and we have characterized its qualitative features. In this section, we analyze the effect of changes in some parameters on some of the key equilibrium objects. In particular, we want to analyze the effect of changes in the menu cost $c$ and in the inflation rate $\pi$ on the cutoff prices $s$ and $S$, on the traveling times $T_{1}$ and $T_{2}$, and on the price distributions $F$ and $G$.

A $(Q, S, s)$ equilibrium exists if and only if the menu cost $c$ belongs to the interval $(0, \bar{c})$, where $\bar{c}$ is a strictly positive number that depends on the value of the other parameters. As we increase the menu cost $c$ in the interval $(0, \bar{c})$, the lowest price $s$ decreases and the lowest new price $S$ increases. Intuitively, $s$ decreases because the marginal cost of deferring a nominal price adjustment increases with the menu cost $c$. Similarly, $S$ increases because the marginal benefit of setting a higher nominal price increases with the menu cost $c$. From these observations, it follows that an increase in the menu cost leads to an increase in the extent of price dispersion, as measured by the range of equilibrium prices $Q-s$,

\footnotetext{
${ }^{2}$ Caplin and Spulber (1987) argue that monetary shocks are neutral when the price distribution is log-uniform. Monetary shocks become less and less neutral when the price distribution has fewer firms close to the lowest price $s$ and more firms close to the highest price $S$. When sellers are subject to large and frequent idiosyncratic shocks, they are more likely to reset their nominal price before reaching the lowest price $s$. In steady-state, this behavior implies that the density of the price distribution is lower at $s$ than at $S$. When the menu costs are larger, the distance between $S$ and $s$ increases and sellers are even more likely to reset their price before reaching the lowest price $s$ and, hence, the density of the price distribution at $\mathrm{s}$ is even lower.
} 
it leads to an increase in the extent of price stickiness, as measured by the maximum duration $T_{1}+T_{2}$ of a nominal price, and it leads to a decline in the contribution of search frictions to price stickiness, as measured by the ratio $T_{1} /\left(T_{1}+T_{2}\right)$.

As the menu cost $c$ approaches $\bar{c}$, the $(Q, S, s)$ equilibrium converges to the equilibrium of a standard $(S, s)$ model. In the limit for $c \rightarrow \bar{c}$, as in a standard $(s, S)$ model, the lowest new price $S$ equals the highest price $Q$, the new price distribution $G$ is degenerate at $Q$, and the price distribution $F$ is log-uniform. Similarly, as the menu cost $c$ approaches 0 , the $(Q, S, s)$ equilibrium converges to the equilibrium of a standard search-theoretic model of price dispersion. In the limit for $c \rightarrow \bar{c}$, as in a standard search model of price dispersion, the lowest price $s$ equals the lowest new price $S$ and the price distribution $F$ is such that seller's profit is the same at any price on the support of the distribution. Notice that, even in the limit for $c \rightarrow 0$, nominal prices are sticky because sellers keep the nominal price constant until its real value reaches $s$. However, none of the nominal price stickiness is caused by menu costs because sellers enjoy exactly the same profits as the real value of their nominal price falls down to $s .^{3}$

A $(Q, S, s)$ equilibrium exists if and only if the inflation rate $\pi$ belongs to the interval $(0, \bar{\pi})$, where $\bar{\pi}$ is a strictly positive number that depends on the value of the other parameters. As we increase the inflation rate $\pi$ in the interval $(0, \bar{\pi})$, the lowest price $s$ remains unchanged and the lowest new price $S$ increases. Intuitively, $s$ does not change because neither the marginal cost nor the marginal benefit of deferring a nominal price adjustment depends on the inflation rate $\pi$. In contrast, $S$ increases because the marginal benefit of setting a higher nominal price increases with the inflation rate $\pi$. Moreover, we find that an increase in the inflation rate has no effect on the extent of price dispersion, as measured by $Q-s$, it leads to a decline in the extent of price stickiness, as measured by $T_{1}+T_{2}$, and it leads to an increase in the contribution of menu costs to price stickiness, as measured by $T_{2} /\left(T_{1}+T_{2}\right)$. When the inflation rate $\pi$ approaches $\bar{\pi}$, the $(Q, S, s)$ equilibrium converges to an $(S, s)$ equilibrium. When the inflation rate $\pi$ approaches 0 ,

\footnotetext{
${ }^{3}$ Head et al. (2012) analyze a version of our model without menu costs. They show that the equilibrium uniquely pins down the distribution of real prices $F$, but it does not uniquely pin down the distribution of new real prices $G$ or the pricing strategy of the individual sellers. They prove that the equilibrium is consistent with nominal price stickiness even though sellers are free to change their price at any time. Moreover, they prove that price stickiness does not cause any monetary non-neutrality. In the limit as the menu cost converges to zero, the equilibrium of our model is the same as in theirs. However, the limit of our model as the menu cost converges to zero uniquely pins down the distribution of new prices $G$ and the pricing strategy of the individual sellers. In this sense, the limit of our model for $c$ going to zero provides a natural refinement of the indeterminate equilibrium objects in Head et al. (2012).
} 
the $(Q, S, s)$ equilibrium does not have any special properties, except that the travelling times $T_{1}$ and $T_{2}$ go to infinity.

The comparative statics results are collected in the following theorem.

Theorem 2: (Comparative statics for $(Q, S, s)$ equilibrium). (i) $A(Q, S, s)$ equilibrium exists iff $c \in(0, \bar{c})$, where $\bar{c}>0$ depends on other parameters. As c increases in $(0, \bar{c}), s$ falls and $S$ increases. Moreover, price dispersion $Q-s$ increases, price stickiness $T_{1}+T_{2}$ increases, and the fraction of price stickiness $T_{1} /\left(T_{1}+T_{2}\right)$ caused by search frictions falls. (ii) $A(Q, S, s)$ equilibrium exists iff $\pi \in(0, \bar{\pi})$, where $\bar{\pi}>0$ depends on other parameters. As $\pi$ increases in $(0, \bar{\pi})$, s does not change and $S$ increases. Moreover, price dispersion $Q-s$ does not change, price stickiness $T_{1}+T_{2}$ falls, and the fraction of price stickiness $T_{1} /\left(T_{1}+T_{2}\right)$ caused by search frictions falls.

Proof: In Appendix B.

\section{$4 \quad(S, s)$ Equilibrium}

An $(S, s)$ equilibrium is an equilibrium where every seller lets inflation erode the real value of its nominal price until it reaches some point $s \in(0, Q)$, then the menu cost is paid and the nominal price changed so that its real value is $S$, where $S=Q$. In subsection 4.1 , we formally define an $(S, s)$ equilibrium. In subsection 4.2 , we derive a necessary and sufficient condition for the existence of an $(S, s)$ equilibrium. Finally, in subsection 4.3 , we characterize the set of parameter values for which an $(S, s)$ equilibrium exists. The main result of this section is that, as long as menu costs are sufficiently small, an $(S, s)$ equilibrium does not exist, but a $(Q, S, s)$ equilibrium does. The result implies that a $(Q, S, s)$ equilibrium is not some odd outcome that exists alongside the more natural $(S, s)$ equilibrium, but that, in fact, a $(Q, S, s)$ equilibrium it is the only possible outcome for some parameter values. ${ }^{4}$

\section{1 $(S, s)$ Equilibrium: Definition}

The problem of an individual seller is the same as in section 3 . That is, the present discounted value of profits for a seller whose nominal price has a real value of $Q \exp (-\pi t)$

\footnotetext{
${ }^{4}$ We do not need to look for equilibria in which a seller resets the real value of its nominal price to $S$, where $S<Q$. Indeed, it is easy to verify that this class of equilibria does not exist because, if $F(S)$ were equal to 1, an individual seller would be strictly better off resetting the real value of its nominal price to $Q$ rather than to $P$. All details are available upon request.
} 
is given by

$$
V(t)=\max _{T}\left\{\int_{t}^{T} e^{-r(x-t)} R\left(Q e^{-\pi x}\right) d x+e^{-r(T-t)}\left(V^{*}-c\right)\right\},
$$

where

$$
R\left(Q e^{-\pi x}\right)=b\left[\alpha+2(1-\alpha)\left(1-F\left(Q e^{-\pi x}\right)\right)\right] Q e^{-\pi x}
$$

The seller finds it optimal to change the nominal price after $T^{*}-x$ units of time, where $T^{*}$ is such that the marginal benefit of deferring a price change, $R\left(Q \exp \left(-\pi T^{*}\right)\right)$, equals the marginal cost of deferring a price change, $r\left(V^{*}-c\right)$. Hence, the seller finds it optimal to change the nominal price when the price's real value is $s$ if and only if $Q \exp \left(-\pi T^{*}\right)=s$ or, equivalently,

$$
R(s)=r\left(V^{*}-c\right)
$$

The seller finds it optimal to reset the real value of the nominal price to $S=Q$ if and only if $V(0)=V^{*}$ and $V(t) \leq V^{*}$ for all $t \in[0, T]$, where $T=\log (S / s) / \pi$ denotes the time it takes for the real value of a nominal price to fall from $S$ to $s$. Using (21), we can write the condition $V(0)=V^{*}$ as

$$
\int_{0}^{T} e^{-r x} R\left(Q e^{-\pi x}\right) d x+e^{-r T}\left(V^{*}-c\right)=V^{*}
$$

Similarly, we can write the condition $V(t) \leq V^{*}$ for all $t \in[0, T]$ as

$$
\int_{t}^{T} e^{-r(x-t)} R\left(Q e^{-\pi x}\right) d x+e^{-r(T-t)}\left(V^{*}-c\right) \leq V^{*} .
$$

In an $(S, s)$ equilibrium, a seller only changes its nominal price when the real value of the price falls down to $s$. Moreover, when a seller changes its nominal price, it always resets it so that the real value of the new price is $S=Q$. As all sellers follow this $(S, s)$ pricing rule, the stationary distribution of real prices is given by

$$
F(p)=\frac{\log (p)-\log (s)}{\log (S)-\log (s)}, \forall p \in[s, S]
$$

The above observations motivate the following definition of equilibrium. 
Definition 2: A stationary $(S, s)$ equilibrium is a $C D F$ of prices $F:[s, Q] \rightarrow[0,1], a$ lower bound on the price distribution $s \in(0, Q)$, an upper bound on the price distribution $S=Q$, and a seller's maximum value $V^{*}$ that jointly satisfy the optimality conditions (23)-(25) and the stationarity condition (26).

\section{2 $(S, s)$ Equilibrium: Existence}

The equilibrium condition (23) states that the profit of a seller with a real price of $s$ must be equal to the annuitized value of paying the menu cost and resetting the nominal price optimally. Since $R(s)$ is given by $b[\alpha+2(1-\alpha)(1-F(s))] s$ and $F(s)$ is equal to 0 , the equilibrium condition (23) implies that the lower bound on the price distribution must be equal to

$$
s=\frac{r\left(V^{*}-c\right)}{b(2-\alpha)}
$$

The equilibrium condition (24) states that the present discounted value of profits for a seller with a real price of $S=Q$ must be equal to the maximum value $V^{*}$. After substituting out $F$ and $s$ and after solving the integral, we can rewrite the equilibrium condition (24) as one equation in the one unknown $V^{*}$, i.e.,

$$
\begin{aligned}
& {\left[\frac{1-e^{-(r+\pi) T\left(V^{*}\right)}\left(1+(r+\pi) T\left(V^{*}\right)\right)}{(r+\pi)^{2}}\right] \frac{2 b(1-\alpha) Q}{T\left(V^{*}\right)}} \\
& +\left[\frac{1-e^{-(r+\pi) T\left(V^{*}\right)}}{r+\pi}\right] b \alpha Q+e^{-r T\left(V^{*}\right)}\left(V^{*}-c\right)-V^{*}=0,
\end{aligned}
$$

where

$$
T\left(V^{*}\right) \equiv \frac{1}{\pi} \log \left(\frac{b(2-\alpha) Q}{r\left(V^{*}-c\right)}\right) .
$$

Clearly, if $\left(F^{*}, S^{*}, s^{*}, V^{*}\right)$ is an $(S, s)$ equilibrium, then $V^{*}$ must be a solution to (28). Conversely, suppose that $V^{*}$ is a solution to $(28)$, with $V^{*} \in(c, b \alpha Q / r]$. Let the upper bound on the price distribution $S^{*}$ be given by $Q$. Let the lower bound on the price distribution $s^{*}$ be given by the solution to $(27)$ for $V=V^{*}$. Let the stationary price distribution $F^{*}$ be given by (26) for $s=s^{*}$ and $S=S^{*}$. Notice that $s^{*}$ is strictly positive as $V^{*}>c$ and that $s^{*}$ is strictly less than $S^{*}$ as $s^{*}<Q$. Notice that $F^{*}$ is a proper cumulative distribution function with support $\left[s^{*}, S^{*}\right]$ as $F^{*}\left(s^{*}\right)=0, F\left(S^{*}\right)=1$ and $F^{* \prime}(p)>0$ for all $p \in\left[s^{*}, S^{*}\right]$. By construction, the tuple $\left(F^{*}, S^{*}, s^{*}, V^{*}\right)$ satisfies the optimality conditions (23)-(24) and the stationarity condition (26). Further, it can be 
proved that, if $V^{*} \leq b \alpha Q / r$, the tuple $\left(F^{*}, S^{*}, s^{*}, V^{*}\right)$ satisfies the optimality condition (25), i.e. the present discounted value of profits for a seller with a real price lower than $S^{*}$ is non-greater than the maximum value $V^{*}$. Therefore, the tuple $\left(F^{*}, S^{*}, s^{*}, V^{*}\right)$ constitutes an $(S, s)$ equilibrium.

If (28) does not admit a solution for $V^{*} \in(c, b \alpha Q / r]$, then an $(S, s)$ equilibrium does not exist. In fact, there is no $(S, s)$ equilibrium associated with a solution $V^{*}$ to equation (28) such that $V^{*} \leq c$. This follows from the fact that $V^{*} \leq c$ implies $s \leq 0$, i.e. the seller finds it optimal to pay the menu cost when the real value of its nominal price becomes negative, an event that never happens. Similarly, there is no $(S, s)$ equilibrium associated with a solution $V^{*}$ to equation (28) such that $V^{*}>b \alpha Q / r$. This follows from the fact that $V^{*}>b \alpha Q / r$ implies $V^{\prime}(0)>0$, i.e. the seller finds it optimal to reset the real value of its nominal price to some $p$ strictly smaller than $S=Q$.

The above observations are formalized in the following theorem.

Theorem 3: (Existence of an $(S, s)$ equilibrium). An $(S, s)$ equilibrium exists if and only if equation (27) admits a solution $V^{*} \in(c, b \alpha Q / r]$.

Proof: In Appendix C.

\section{3 $(S, s)$ Equilibrium: Comparative Statics}

Theorem 3 states that an $(S, s)$ equilibrium exists if and only if there exists a $V^{*}$ such that: (i) $V^{*}$ is a solution to (28); (ii) $V^{*}$ is greater than $c$ and smaller than $b \alpha Q / r$. It is convenient to rewrite these two conditions in terms of $x=V^{*}-c$ rather than $V^{*}$. Condition (i) is equivalent to $x$ being a solution to

$$
\begin{aligned}
& {\left[\frac{1-e^{-(r+\pi) T(x)}(1+(r+\pi) T(x))}{(r+\pi)^{2}}\right] \frac{2 b(1-\alpha) Q}{T(x)}} \\
& +\left[\frac{1-e^{-(r+\pi) T(x)}}{r+\pi}\right] b \alpha Q+e^{-r T(x)} x-x=c,
\end{aligned}
$$

where

$$
T(x) \equiv \frac{1}{\pi} \log \left(\frac{b(2-\alpha) Q}{r x}\right) .
$$

Similarly, condition (ii) is equivalent to $x$ being positive and such that

$$
\frac{b \alpha Q}{r}-x \geq c
$$




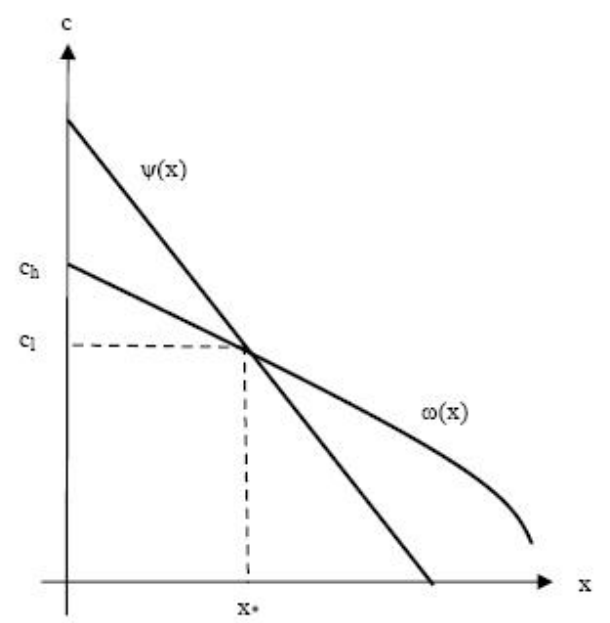

(a) Example 1

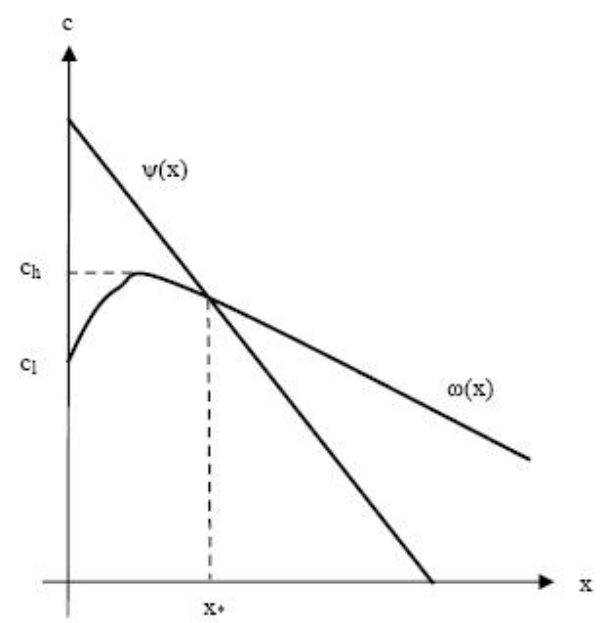

(b) Example 2

Figure 5: Existence of an $(S, s)$ equilibrium

Now, let $\omega(x)$ denote the left-hand side of (29). It is straightforward to verify that the function $\omega(x)$ is such that $\omega(0)=b \alpha Q /(r+\pi), \omega^{\prime}(x)>-1$ and $\omega(x)>0$ for all $x \in[0, b \alpha Q / r]$. Similarly, let $\psi(x)$ denote the left-hand side of (30). Clearly, the function $\psi(x)$ is such that $\psi(0)=b \alpha Q / r, \psi(b \alpha Q / r)=0$ and $\psi^{\prime}(x)=-1$. Figure 5 illustrates the properties of $\omega(x)$ and $\psi(x)$ in the $(x, c)$ space.

The properties of $\omega(x)$ and $\psi(x)$ imply that there exists an $x^{*} \in(0, b \alpha Q / r)$ such that $\omega(x)<\psi(x)$ for all $x \in\left[0, x^{*}\right), \omega(x)=\psi(x)$ for $x=x^{*}$, and $\omega(x)>\psi(x)$ for all $x \in\left(x^{*}, b \alpha Q / r\right]$. Let $c_{h}$ denote the maximum of the function $\omega(x)$ over the interval $\left[0, x^{*}\right]$. Notice that, since $\omega(0)<\psi(0), c_{h}$ is strictly smaller than $b \alpha Q / r$. Similarly, let $c_{\ell}$ denote the minimum of the function $\omega(x)$ over the interval $\left[0, x^{*}\right]$. Notice that, since $\omega(x)>0$ for all $x \in[0, b \alpha Q / r], c_{\ell}$ is strictly greater than zero. Figure 5 illustrates the definition of $x^{*}, c_{\ell}$ and $c_{h}$.

The following observations follow immediately from the inspection of Figure 5. If the menu cost $c$ is smaller than $c_{\ell}$, an $(S, s)$ equilibrium cannot exist as there is no $x$ such that $\omega(x)=c$ and $\psi(x) \leq c$. Similarly, if the menu cost $c$ is greater than $c_{h}$, an $(S, s)$ equilibrium cannot exist as there is no $x$ such that $\omega(x)=c$ and $\psi(x) \leq c$. In contrast, if the menu cost $c$ is greater than $c_{\ell}$ and smaller than $c_{h}$, an $(S, s)$ equilibrium exists as there exists at least one $x$ such that $\omega(x)=c$ and $\psi(x) \leq c$. 
When the menu cost $c$ is between $c_{\ell}$ and $c_{h}$, the $(S, s)$ equilibrium need not be unique because the function $\omega(x)$ may not be monotonic. This case is illustrated in panel (b) of Figure 5. Intuitively, multiple $(S, s)$ equilibria may exist because of a feed-back effect between the stationary distribution of prices, $F$, and the seller's maximum value, $V^{*}$. The higher is the seller's maximum value, the lower is the point $s$ at which a seller finds it optimal to reset its nominal price and, hence, the lower is the stationary distribution of prices $F$. Conversely, the lower is the stationary distribution of prices $F$, the stronger is the competition faced by a seller offering any particular price and, hence, the lower is the seller's maximum value $V^{*}$. For some parameter values, this feed-back effect is so strong as to generate multiple $(S, s)$ equilibria.

Finally, note that the interval of menu costs for which an $(S, s)$ equilibrium exists, i.e. $\left[c_{\ell}, c_{h}\right]$, always contains the upper bound of the interval of menu costs for which a $(Q, S, s)$ equilibrium exists, i.e. $\bar{c}$. For $c=\omega\left(x^{*}\right)$, there exists an $(S, s)$ equilibrium in which the maximum value of the seller is $b \alpha Q / r$. For $c \rightarrow \bar{c}$, the $(Q, S, s)$ equilibrium converges to an $(S, s)$ equilibrium in which the maximum value of the seller is $b \alpha Q / r$. Therefore, $\bar{c}$ is equal to $\omega\left(x^{*}\right) \in\left[c_{\ell}, c_{h}\right]$.

The above results are summarized in the following theorem.

Theorem 4: (Comparative statics for $(S, s)$ equilibrium). For any menu cost $c$ in the interval $\left[c_{\ell}, c_{h}\right]$, there exists at least one $(S, s)$ equilibrium. The bounds $c_{\ell}$ and $c_{h}$ are such that $c_{\ell}>0, c_{h}<b \alpha Q / r$ and $\bar{c} \in\left[c_{\ell}, c_{h}\right]$.

The key result in Theorem 4 is that an $(S, s)$ equilibrium cannot exist if the menu cost is sufficiently small. There is a simple intuition for this result. When the menu cost is smaller, the lower bound $s$ on the price distribution is higher as the seller's marginal cost of deferring a price adjustment, $r\left(V^{*}-c\right)$, increases relative to the marginal benefit, $R(s)=b(2-\alpha) s$. Consequently, when the menu cost is smaller, the stationary price distribution $F$ becomes more compressed towards the upper bound $S$. When the menu cost is sufficiently small, the price distribution become so compressed at the top that an individual seller is better off deviating from the equilibrium by resetting the real value of its nominal price to some $p$ smaller than $S=Q$. The seller finds the deviation profitable because, by resetting its price to $p$ rather than to $Q$, it can sell not only to the buyers who have not met any other seller, but also to the many buyers who have met a seller charging a price between $p$ and $Q$. Thus, when the menu is sufficiently low, an $(S, s)$ equilibrium with $S=Q$ does not exists. 
Could there exist an $(S, s)$ equilibrium with $S<Q$ ? The answer is negative. To see this suppose for a moment that there exists an $(S, s)$ equilibrium in which every seller resets its price to some $S$ smaller than $Q$. In this equilibrium, a seller's present discounted value of profits must be greater at $S$ than at any lower price. This condition can be satisfied if the seller's value at $S$ is some $V^{*}$ smaller than $b \alpha S / r$. However, if $V^{*} \leq b \alpha S / r$, an individual seller is better off deviating from the equilibrium by resetting the real value of its nominal price to $Q$. Intuitively, the seller find this deviation profitable because, by resetting its price to $Q$ rather than to $S$, it still only sells to the buyers who have not met any other seller but it can enjoy a higher profit margin. More precisely, by resetting its price to $Q$ rather than to $S$, the seller can enjoy a period of length $\log (Q / S) / \pi$ during which the flow profit is strictly greater than $b \alpha S$. As $b \alpha S \geq r V^{*}$, the seller can attain a higher present discounted value of profits. Thus, an $(S, s)$ equilibrium with $S<Q$ does not exist.

Overall, when the menu cost is sufficiently low, there cannot be an $(S, s)$ equilibrium with $S=Q$ as an individual seller would want to deviate from this equilibrium by resetting its price below $S$. Moreover, there cannot be an $(S, s)$ equilibrium with $S<Q$ as an individual seller would want to deviate from this equilibrium by resetting its price above $S$ (to $Q$ ). The natural resolution of this tension is an equilibrium in which sellers randomize with respect to their reset price: a $(Q, S, s)$ equilibrium. As it is clear from the discussion in the previous paragraphs, the economic forces that rule out an $(S, s)$ equilibrium and ask for an equilibrium in which sellers randomize with respect to their reset price are exactly the same economic forces that, absent menu costs, rule out a unique price equilibrium and ask for an equilibrium in which sellers randomize with respect to their price.

Theorem 2 guarantees that there are no holes between the region of parameters for which an $(Q, S, s)$ equilibrium exists and the region of parameters for which an $(S, s)$ equilibrium exists. However, Theorem 2 does not tell us whether the $(Q, S, s)$ and the $(S, s)$ existence regions overlap or not. Figure 6 shows that the answer depends on parameter values. Figure 6(a) shows the combinations of menu costs, $c$, and inflation rates, $\pi$, for which there exists a $(Q, S, s)$ equilibrium and for which there exists an $(S, s)$ equilibrium, given that the parameters $(\alpha, r, b, Q)$ take on the values $(.5, .03,1,1)$. In particular, a $(Q, S, s)$ equilibrium exists for combinations of $c$ and $\pi$ in the dark gray area between the thin black line and the $x$-axis. Similarly, an $(S, s)$ equilibrium exists for combinations of $c$ and $\pi$ in the dark gray area between the dashed red line and the blue line. In this example, the $(Q, S, s)$ and the $(S, s)$ existence regions are non-overlapping: if $c \leq c_{\ell}=\bar{c}$, 


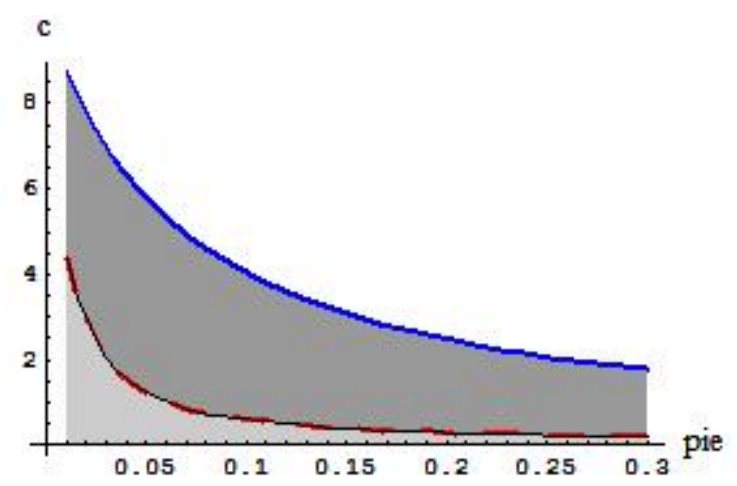

(a) Example 1

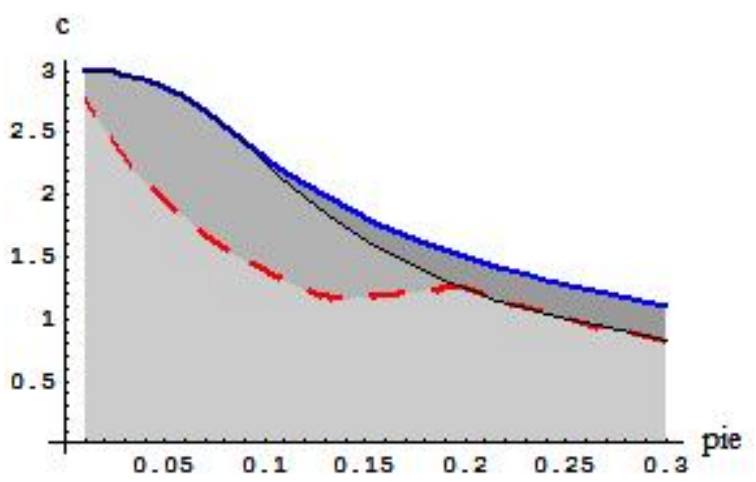

(b) Example 2

Figure 6: Existence regions

only a $(Q, S, s)$ equilibrium exists; if $c \in\left[c_{\ell}, c_{h}\right]$, only an $(S, s)$ equilibrium exists; and if $c>c_{h}$, neither type of equilibrium exists. ${ }^{5}$ Figure $6(\mathrm{~b})$ shows the $(Q, S, s)$ and $(S, s)$ existence regions when the parameters $(\alpha, r, b, Q)$ take on the values $(.15, .03,1,1)$. In this example, the two existence regions are overlapping: if $c<c_{\ell}$, only a $(Q, S, s)$ equilibrium exists; if $c \in\left[c_{\ell}, c_{h}\right)$, both a $(Q, S, s)$ and an $(S, s)$ equilibrium exist; if $c \in\left[\bar{c}, c_{h}\right]$, only an $(S, s)$ equilibrium exists; and if $c>c_{h}$, neither type of equilibrium exists.

\section{Conclusions}

In this paper, we studied the effect of menu costs on the pricing behavior of sellers and on the cross-sectional distribution of prices in a search-theoretic model of imperfect competition in the spirit of Butters (1977), Varian (1980) or Burdett and Judd (1983). The distinguishing aspect of our model of imperfect competition is that, even without menu costs, the equilibrium features a non-degenerate distribution of prices. We proved that, when the menu cost is sufficiently small, the only equilibrium is a $(Q, S, s)$ equilibrium, i.e. an equilibrium in which sellers let inflation erode the real value of their price until it reaches some point $s$, then they pay the menu cost and change their nominal price so that the real value of the new price is randomly drawn from a distribution with support $[S, Q]$, where $Q$ is the buyer's reservation price and $S$ is some price between $s$ and $Q$. Then, we

\footnotetext{
${ }^{5}$ When $c>c_{h}$, a stationary equilibrium does not exist because the menu cost $c$ is greater than the seller's maximum value $V^{*}$ and, hence, sellers never have the incentive to adjust their nominal price. However, if we were to let sellers decide whether to enter the market at some cost $k>0$, we would find an $(S, s)$ equilibrium with a higher buyer-to-seller ratio $b$.
} 
established several features of a $(Q, S, s)$ equilibrium. The seller's present value of profits is constant as the real price falls from $Q$ to $S$, and it is monotonically decreasing as the real price falls from $S$ to $s$. The seller's flow profit is constant as the real price falls from $Q$ to $S$, and it is first increasing and then decreasing as the real price falls from $S$ to $s$. Over the interval $[S, Q]$, the cross-sectional distribution of prices is such that the seller's flow profit is constant. Over the interval $[s, S]$, the cross-sectional distribution of prices is log-uniform. Overall, the model behaves like a standard search model of price dispersion for prices between $S$ and $Q$ (see, e.g., BJ83) and like a standard menu cost model for prices between $s$ and $S$ (see, e.g., B88). Finally, we explained that, when the menu cost is sufficiently small, there is a $(Q, S, s)$ equilibrium but not an $(S, s)$ equilibrium for exactly the same reasons why, in the absence of menu costs, the equilibrium features price dispersion. This last observation makes us conjecture that, for small menu costs, a $(Q, S, s)$ equilibrium will emerge also in other models that, in the absence of menu costs, feature price dispersion (see, e.g., Prescott 1975 and Eden 1994).

There are several applications of our model that seem promising. Just two are mentioned below. First, it should be possible to estimate the model using data on the distribution of nominal prices across sellers and data on the evolution of nominal prices at each individual sellers. The exercise would allow us to estimate both search frictions and menu costs and to quantify their relative contribution to price stickiness. Second, it should be possible to extend the model to allow for seller-specific shock and estimate it using data on the distribution of nominal prices across sellers, on the distribution of nominal price changes and on the duration of individual prices. The exercise would allow us to assess the magnitude of menu costs and seller-specific shocks and, in turn, to measure the real effect of monetary shocks in a search-theoretic model of imperfect competition.

\section{References}

[1] Benabou R. 1988. "Search, Price Setting and Inflation." Review of Economic Studies, 55: $353-376$.

[2] Benabou R. 1992. "Inflation and Efficiency in Search Markets." Review of Economic Studies, 59: 299-329.

[3] Burdett, K., and K. Judd. 1983. "Equilibrium Price Dispersion.” Econometrica, 51: 955-970. 
[4] Butters, G. 1977. "Equilibrium Distributions of Sales and Advertising Prices." Review of Economic Studies, 44: 465-491.

[5] Caplin, A., and J. Leahy. 1991. "State-Dependent Pricing and the Dynamics of Money and Output." Quarterly Journal of Economics, 107: 683-708.

[6] Caplin, A., and J. Leahy. 1997. "Aggregation and Optimization with State-Dependent Pricing." Econometrica, 65: 601-625.

[7] Caplin, A., and J. Leahy. 2010. "Economic Theory and the World of Practice: A Celebration of the $(S, s)$ Model." Journal of Economic Perspectives, 24: 183-202.

[8] Caplin, A., and D. Spulber. 1986. "Menu Costs and the Neutrality of Money." Quarterly Journal of Economics, 102: 703-725.

[9] Diamond, P. 1971. "A Model of Price Adjustment." Journal of Economic Theory, 3: 156-168.

[10] Dotsey, M., R. King and A. Wolman. 1999. "State-Dependent Pricing and the General Equilibrium Dynamics of Money and Output." Quarterly Journal of Economics, 114: 655-690.

[11] Eden, B. 1994. "The Adjustment of Prices to Monetary Shocks When Trade is Uncertain and Sequential." Journal of Political Economy.

[12] Golosov, M., and R. Lucas. 2007. "Menu Costs and Phillips Curves." Journal of Political Economy, 115: 171-99.

[13] Head, A., L. Liu, G. Menzio and R. Wright. 2012. "Sticky Prices: A New Monetarist Approach." Journal of the European Economic Association, 10: 939-973.

[14] Hong, H., and M. Schum. 2006. "Using Price Distributions to Estimate Search Costs." Rand Journal of Economics.

[15] Kaplan, G., G. Menzio and M. Wildenbeest. 2013. "Product Market Search and Employment Status." Manuscript, Princeton University.

[16] Klenow, P., and O. Kryvtsov. 2008. "State-Dependent or Time-Dependent Pricing: Does it Matter for Recent U.S. Inflation?" Quarterly Journal of Economics, 123: 863-904. 
[17] Lagos, R., and R. Wright. 2005. "A Unified Framework for Monetary Theory and Policy Analysis." Journal of Political Economy 113: 463-484.

[18] Lucas, R., and N. Stokey. 1987. "Money and Interest in a Cash-in-Advance Economy." Econometrica 55: 491-513.

[19] Midrigan, V. 2011. "Menu Costs, Multi-Product Firms, and Aggregate Fluctuations." Econometrica,79: 1139-1180.

[20] Moraga-Gonzales, J., and M. Wildenbeest. 2008. "Maximum Likelihood Estimation of Search Costs." European Economic Review.

[21] Nakamura, E., and J. Steinsson. 2008. "Five Facts About Prices: A Reevaluation of Menu Cost Models." Quarterly Journal of Economics, 123: 1415-1464.

[22] Prescott, E. 1975. "Efficiency of the Natural Rate." Journal of Political Economy.

[23] Sheshinski, E., and Y. Weiss. 1982. "Inflation and Costs of Price Adjustment." Review of Economic Studies, 44: 287-303.

[24] Varian, H. 1980. "A Theory of Sales." American Economic Review, 70. 


\section{Appendix}

\section{A Proof of Theorem 1}

(i) Part (i) of Theorem 1 states that a $(Q, S, s)$ equilibrium exists if and only if $\varphi(Q)>0$. The function $\varphi(S)$ is defined as

$$
\begin{aligned}
\varphi(S) \equiv & {\left[\frac{1-e^{-(r+\pi) T_{2}(S)}\left(1+(r+\pi) T_{2}(S)\right)}{(r+\pi)^{2}}\right] \frac{(2-\alpha) S-\alpha Q}{T_{2}(S)} } \\
& +\left[\frac{1-e^{-(r+\pi) T_{2}(S)}}{r+\pi}\right] \alpha Q+e^{-r T_{2}(S)}\left(\frac{\alpha Q}{r}-\frac{c}{b}\right)-\frac{\alpha Q}{r}=0
\end{aligned}
$$

where

$$
T_{2}(S) \equiv \frac{\log (S / s)}{\pi}, \quad s=\frac{\alpha Q-r c / b}{2-\alpha} .
$$

It is straightforward to verify that $\varphi(S)$ has the following properties: (i) $\varphi(S)<0$ for all $S \in[s, \alpha Q /(2-\alpha)]$; (ii) $\varphi^{\prime}(S)>0$ for all $S \in[\alpha Q /(2-\alpha), Q]$.

Suppose that $\varphi(Q) \leq 0$. Then a $(Q, S, s)$ equilibrium does not exist. On the way to a contradiction, let $(F, G, S, s, V)$ be a $(Q, S, s)$ equilibrium. As proved in Section 3 , if $(F, G, S, s, V)$ is a $(Q, S, s)$ equilibrium, then $S$ must be a solution to the equation $\varphi(S)=0$ and it must belong to the interval $(s, S)$. However, $\varphi(S)=0$ cannot admit any solution in the interval $(s, Q)$ because $\varphi(S)<0$ for all $S \in(s, Q)$.

Conversely, suppose that $\varphi(Q)>0$. Then, the equation $\varphi(S)=0$ admits at most one solution in the interval $(s, Q)$ because $\varphi(s)<0$ and $\varphi(Q) \leq 0$ and $\varphi(S)$ is strictly increasing in $S$. Let $S^{*}$ denote this solution. Let $s^{*}$ be defined as in equation (19) for $S=S^{*}$. Let $F^{*}$ be defined as in equations (14) and (15) for $S=S^{*}$ and $s=s^{*}$. Let $G^{*}$ be defined as in equations (16)-(18) for $S=S^{*}$ and $s=s^{*}$. Let $V^{*}$ be defined as in equation (13). Moreover, let $T_{1}^{*}$ and $T_{2}^{*}$ be defined, respectively, as $\log \left(Q / S^{*}\right) / \pi$ and $\log \left(S^{*} / s^{*}\right) / \pi$ and let $R(p)$ be defined as in (2) for $F=F^{*}$. To prove that the tuple $\left(F^{*}, G^{*}, S^{*}, s^{*}, V^{*}\right)$ constitutes a $(Q, S, s)$ equilibrium, we need to verify that it jointly satisfies the optimality conditions (4)-(7) and the stationarity conditions (9), (11) and (12). In addition, we need to verify that $S^{*} \in\left(s^{*}, Q\right), s^{*} \in[0, Q), F^{*}$ is a CDF with support $\left[s^{*}, Q\right]$ and $G^{*}$ is a CDF with support $\left[S^{*}, Q\right]$.

The tuple $\left(F^{*}, G^{*}, S^{*}, s^{*}, V^{*}\right)$ satisfies the stationarity condition (9) because, for all 
$p \in\left(s^{*}, S^{*}\right)$, we have

$$
\begin{aligned}
F^{* \prime}(p) p & \equiv\left[1-\frac{\alpha}{2(1-\alpha)} \frac{Q-S^{*}}{S^{*}}\right] \frac{1}{\log \left(S^{*} / s^{*}\right)} \\
& =F^{* \prime}\left(s^{*}\right) s^{*}
\end{aligned}
$$

Similarly, the stationarity condition (11) is satisfied because, for all $p \in\left(S^{*}, Q\right)$, we have

$$
\begin{aligned}
F^{* \prime}(p) p & \equiv \frac{\alpha}{2(1-\alpha)} \frac{Q}{p} \\
& =\left[1-\frac{\alpha}{2(1-\alpha)} \frac{Q-S^{*}}{S^{*}}\right]^{-1}\left[1-\frac{\alpha}{2(1-\alpha)} \frac{Q-S^{*}}{S^{*}}\right] \frac{s^{*}}{\log \left(S^{*} / s^{*}\right)} \frac{\alpha \log \left(S^{*} / s^{*}\right) Q}{2(1-\alpha) s^{*} p} \\
& =F^{* \prime}(s)\left(1-G^{*}(p)\right) s^{*}
\end{aligned}
$$

Moreover, notice that $F^{*}$ is a CDF with support $\left[s^{*}, Q\right]$. In fact, $F^{*}\left(s^{*}\right)=0, F^{*}(Q)=1$ and $F^{* \prime}(p)>0$ for all $p \in\left[s^{*}, Q\right]$.

The tuple $\left(F^{*}, G^{*}, S^{*}, s^{*}, V^{*}\right)$ satisfies the optimality condition (4) because

$$
\begin{aligned}
r\left(V^{*}-c\right) & =b \alpha Q-c r \\
& =b\left[\alpha+2(1-\alpha)\left(1-F^{*}\left(s^{*}\right)\right)\right] s^{*} \\
& =R\left(s^{*}\right) .
\end{aligned}
$$

The optimality condition (5) is satisfied because, for all $t \in\left[0, T_{1}^{*}\right]$, we have

$$
\begin{aligned}
R\left(Q e^{-\pi t}\right) & =b\left[\alpha+2(1-\alpha)\left(1-F^{*}\left(Q e^{-\pi t}\right)\right)\right] Q e^{-\pi t} \\
& =b \alpha Q=r V^{*}
\end{aligned}
$$

The optimality condition (6) is satisfied because $\varphi\left(S^{*}\right)=0$ implies

$$
\int_{T_{1}^{*}}^{T_{1}^{*}+T_{2}^{*}} e^{-r\left(x-T_{1}^{*}\right)} R\left(Q e^{-\pi x}\right) d x+e^{-r T_{2}^{*}}\left(V^{*}-c\right)-V^{*}=0 .
$$

Moreover, $S^{*} \in\left(s^{*}, Q\right)$ and the assumption $c \in(0, b \alpha Q / r)$ implies

$$
s^{*} \equiv \frac{\alpha Q-r c / b}{2-\alpha} \in(0, Q)
$$

The optimality condition (5) together with the optimality condition (6) guarantees that the seller's value $V(t)$ is equal to $V^{*}$ for all $t \in\left[0, T_{1}^{*}\right]$. Now, we need to verify that the optimality condition (7) That is, we need to verify that the seller's value $V(t)$ is 
non-greater than $V^{*}$ for all $t \in\left[T_{1}^{*}, T_{1}^{*}+T_{2}^{*}\right]$ To this aim, notice that $V(t)$ satisfies the differential equation

$$
\begin{aligned}
& r V(t)=\hat{R}(t)+V^{\prime}(t), \\
& \hat{R}(t) \equiv R\left(Q e^{-\pi t}\right) .
\end{aligned}
$$

Notice that, for $t \in\left[T_{1}^{*}, T_{1}^{*}+T_{2}^{*}\right], \hat{R}(t)$ is given by

$$
\hat{R}(t)=e^{-\pi\left(t-T_{1}^{*}\right)} b\left\{(2-\alpha) S^{*}-\left[(2-\alpha) S^{*}-\alpha Q\right]\left[1-\frac{\pi\left(t-T_{1}^{*}\right)}{\log \left(S^{*} / s^{*}\right)}\right]\right\} .
$$

The derivative of $\hat{R}(t)$ with respect to $t$ is given by

$$
\hat{R}^{\prime}(t)=\pi e^{-\pi\left(t-T_{1}^{*}\right)} b\left\{\left[(2-\alpha) S^{*}-\alpha Q\right]\left[1+\frac{1-\pi\left(t-T_{1}^{*}\right)}{\log \left(S^{*} / s^{*}\right)}\right]-(2-\alpha) S^{*}\right\} .
$$

Notice that $\hat{R}^{\prime}(t)$ has the same sign as

$$
\sigma(t)=\left[(2-\alpha) S^{*}-\alpha Q\right]\left[1+\frac{1-\pi\left(t-T_{1}^{*}\right)}{\log \left(S^{*} / s^{*}\right)}\right]-(2-\alpha) S^{*} .
$$

It is straightforward to verify that $\sigma(t)$ is strictly decreasing in $t$ for all $t \in\left[T_{1}^{*}, T_{1}^{*}+T_{2}^{*}\right]$ and that $\sigma\left(T_{1}^{*}\right)$ is strictly positive (negative) if $(2-\alpha) S^{*}$ is strictly greater (smaller) than $\alpha Q\left(1+\log \left(S^{*} / s^{*}\right)\right)$. Therefore, if $(2-\alpha) S^{*}>\alpha Q\left(1+\log \left(S^{*} / s^{*}\right)\right), \hat{R}(t)$ is first strictly increasing and then strictly decreasing over the interval $\left[T_{1}^{*}, T_{1}^{*}+T_{2}^{*}\right]$. If $(2-\alpha) S^{*}<$ $\alpha Q\left(1+\log \left(S^{*} / s^{*}\right)\right), \hat{R}(t)$ is strictly decreasing for all $t \in\left[T_{1}^{*}, T_{1}^{*}+T_{2}^{*}\right]$. Notice that $\hat{R}(t)$ cannot be increasing for all $t \in\left[T_{1}^{*}, T_{1}^{*}+T_{2}^{*}\right]$ because $\hat{R}\left(T_{1}^{*}\right)=b \alpha Q$ and $\hat{R}\left(T_{1}^{*}+T_{2}^{*}\right)=$ $b \alpha Q-c r$.

Consider the phase diagram in Figure 7, which describes the differential equation (A9). The black line passing through the origin denotes the locus of points $(\hat{R}, V)$ such that $V=\hat{R} / r$ and, hence, $V^{\prime}=0$. Any point below the black line is such that $V<\hat{R} / r$ and, hence, $V^{\prime}<0$. Any point above the black line is such that $V>\hat{R} / r$ and, hence, $V^{\prime}>0$. From (5) and (6), it follows that $\hat{R}\left(T_{1}^{*}\right)=b \alpha Q$ and $V\left(T_{1}^{*}\right)=b \alpha Q / r$. Hence, the point $\left(\hat{R}\left(T_{1}^{*}\right), V\left(T_{1}^{*}\right)\right)$ lies on the black line and $V^{\prime}\left(T_{1}^{*}\right)=0$. From (4) and (13), it follows that $\hat{R}\left(T_{1}^{*}+T_{2}^{*}\right)=b \alpha Q-c r$ and $V\left(T_{1}^{*}+T_{2}^{*}\right)=b \alpha Q / r-c$. Hence, the point $\left(\hat{R}\left(T_{1}^{*}+T_{2}^{*}\right), V\left(T_{1}^{*}+T_{2}^{*}\right)\right)$ lies on the black line and $V^{\prime}\left(T_{1}^{*}+T_{2}^{*}\right)=0$.

Now, we want to find out the trajectory that the pair $(\hat{R}(t), V(t))$ follows as it goes from the point $(b \alpha Q, b \alpha Q / r)$ at $t=T_{1}^{*}$ to the point $(b \alpha Q-c r, b \alpha Q / r-c)$ at $t=T_{1}^{*}+T_{2}^{*}$. Notice that $(2-\alpha) S^{*}$ must be greater than $\alpha Q\left(1+\log \left(S^{*} / s^{*}\right)\right)$. In fact, if $(2-\alpha) S \leq$ 


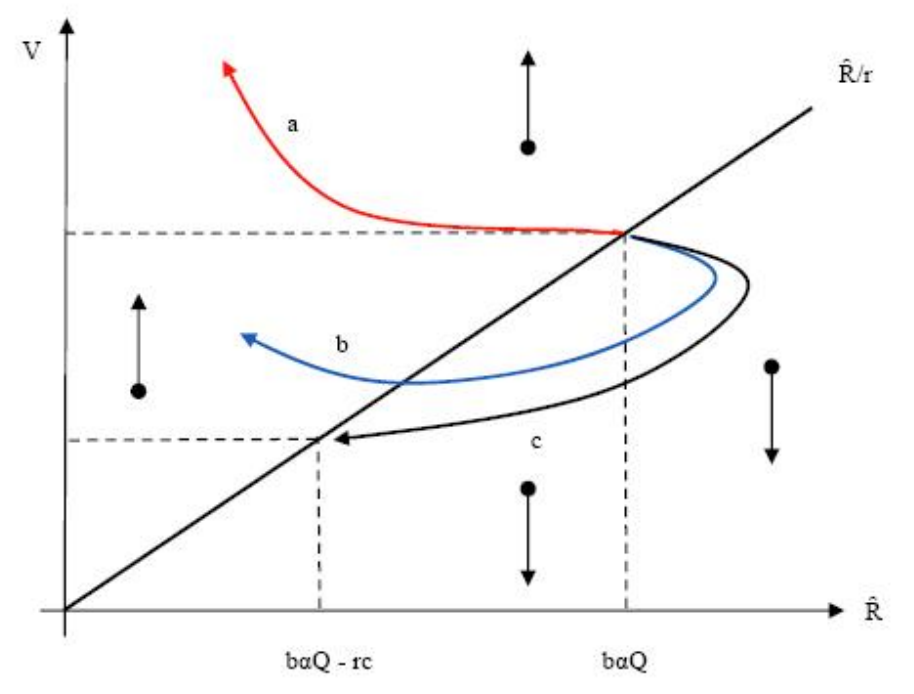

Figure 7: Joint Dynamics of $V(t)$ and $\hat{R}(t)$

$\alpha Q\left(1+\log \left(S^{*} / s^{*}\right)\right), \hat{R}(t)$ is strictly decreasing for all $t \in\left[T_{1}^{*}, T_{1}^{*}+T_{2}^{*}\right]$. As illustrated by the trajectory (a) in the phase diagram, this implies that $(\hat{R}(t), V(t))$ exits the initial point $(b \alpha Q, b \alpha Q / r)$ from the left, enters the region where $V^{\prime}(t)>0$, and remains in that region for all $t \in\left(T_{1}^{*}, T_{1}^{*}+T_{2}^{*}\right)$. Thus, $V\left(T_{1}^{*}+T_{2}^{*}\right)>V\left(T_{1}^{*}\right)=b \alpha Q / r$, which contradicts the fact that $V\left(T_{1}^{*}+T_{2}^{*}\right)=b \alpha Q / r-c$.

Since $(2-\alpha) S>\alpha Q\left(1+\log \left(S^{*} / s^{*}\right)\right), \hat{R}(t)$ is first increasing and then decreasing over the interval $\left[T_{1}^{*}, T_{1}^{*}+T_{2}^{*}\right]$. As illustrated by trajectories (b) and (c) in the phase diagram, this implies that $(\hat{R}(t), V(t))$ exits the initial point $(b \alpha Q, b \alpha Q / r)$ from the right, enters the region where $V^{\prime}(t)<0$, and remains in that region until it crosses the black line either at some $\hat{t}<T_{1}^{*}+T_{2}^{*}$, as in trajectory (b), or at $T_{1}^{*}+T_{2}^{*}$, as in trajectory (c). If $(\hat{R}(t), V(t))$ crosses the black line at some $\hat{t}<T_{1}^{*}+T_{2}^{*}$, then $\hat{R}^{\prime}(\hat{t})<0$. This implies that $V^{\prime}(t)>0$ and $\hat{R}^{\prime}(t)<0$ for all $t \in\left(\hat{t}, T_{1}^{*}+T_{2}^{*}\right)$. Thus, $(\hat{R}(t), V(t))$ cannot reach the end point $(b \alpha Q-c r, b \alpha Q / r-c)$. Therefore, the pair $(\hat{R}(t), V(t))$ must follow the trajectory (c). Along this trajectory, $V(t)$ is strictly decreasing.

To complete the existence proof, we still need to verify that $G^{*}$ is a CDF with support 
$\left[S^{*}, Q\right]$. To this aim, recall that $G^{*}$ is given by

$$
G^{*}(p) \equiv \begin{cases}0, & \text { if } p<S^{*}, \\ 1-\left[1-\frac{\alpha}{2(1-\alpha)} \frac{Q-S^{*}}{S^{*}}\right]^{-1} \frac{\alpha \log \left(S^{*} / s^{*}\right) Q}{2(1-\alpha) p}, & \text { if } p \in\left[S^{*}, Q\right), \\ 1, & \text { if } p \geq Q .\end{cases}
$$

The distribution function $G^{*}(p)$ has the following properties: (i) $G^{*}(p)=0$ for all $p<S^{*}$; (ii) $G^{*}\left(S^{*}\right) \geq 0$ if and only if $(2-\alpha) S^{*} \geq \alpha Q\left[1+\log \left(S^{*} / s^{*}\right)\right]$; (iii) $G^{* \prime}(p)>0$ for all $p \in\left(S^{*}, Q\right)$ if and only if $S^{*}>\alpha Q /(2-\alpha)$; (iv) $G^{*}(Q-) \leq 1$ if and only if $S^{*}>\alpha Q /(2-\alpha)$; (iv) $G^{*}(p)=1$ for all $p \geq Q$. Therefore, $G^{*}(p)$ is a proper CDF with support $\left[S^{*}, Q\right]$ if and only if $S^{*}>\alpha Q /(2-\alpha)$ and $(2-\alpha) S^{*} \geq \alpha Q\left[1+\log \left(S^{*} / s^{*}\right)\right]$. We have already established that both conditions hold.

(ii) Part (ii) of Theorem 1 states that: (a) $V^{\prime}(t)=0$ for all $t \in\left(0, T_{1}^{*}\right)$ and $V^{\prime}(t)<0$ for all $t \in\left(T_{1}^{*}, T_{1}^{*}+T_{2}^{*}\right)$; (b) $\hat{R}^{\prime}(t)=0$ for all $t \in\left(0, T_{1}^{*}\right), \hat{R}^{\prime}(t)>0$ for all $t \in\left(T_{1}^{*}, \hat{T}\right)$ and $\hat{R}^{\prime}(t)<0$ for all $t \in\left(\hat{T}, T_{1}^{*}+T_{2}^{*}\right)$. Both properties have been established while proving part (i).

\section{B Proof of Theorem 2}

(i) In the proof of Theorem 1, we showed that a $(Q, S, s)$ equilibrium exists if and only if the equation $\Phi(S, c)=0$ admits a solution for $S \in(\alpha Q /(2-\alpha), Q)$. The function $\Phi(S, c)$ is given by

$$
\begin{aligned}
\varphi(S, c)= & {\left[\frac{1-e^{-(r+\pi) T_{2}(S, c)}\left(1+(r+\pi) T_{2}(S, c)\right)}{(r+\pi)^{2} T_{2}(S, c)}\right][(2-\alpha) S-\alpha Q] } \\
& +\left[\frac{1-e^{-(r+\pi) T_{2}(S, c)}}{r+\pi}\right] \alpha Q+e^{-r T_{2}(S, c)}\left(\frac{\alpha Q}{r}-\frac{c}{b}\right)-\frac{a Q}{r},
\end{aligned}
$$

where $T_{2}(S, c)$ is given by

$$
T_{2}(S, c)=\frac{\log (S / s(c))}{\pi}, \quad s(c)=\frac{\alpha Q-r c / b}{2-\alpha} .
$$

Let $\varphi_{S}(S, c)$ and $\varphi_{c}(S, c)$ denote the derivatives of $\varphi(S, c)$ with respect to $S$ and $c$. 
The derivative $\varphi_{S}(S, c)$ is given by

$$
\begin{aligned}
& \varphi_{S}(S, c)= \\
& \quad\left[\frac{1-e^{-(r+\pi) T_{2}(S, c)}\left(1+(r+\pi) T_{2}(S, c)\right)}{(r+\pi)^{2}}\right] \frac{(2-\alpha) S[\log (S / s(c))-1]+\alpha Q}{\log (S / s(c))^{2}} .
\end{aligned}
$$

The derivative $\varphi_{c}(S, c)$ is given by

$$
\begin{aligned}
& \varphi_{c}(S, c)=-\frac{e^{-r T_{2}(S, c)}}{b} \\
& -\left[\frac{1-e^{-(r+\pi) T_{2}(S, c)}\left(1+(r+\pi) T_{2}(S, c)\right)}{(r+\pi)^{2}}\right]\left[\frac{(2-\alpha) S-\alpha Q}{b \log (S / s(c))^{2}}\right] \frac{\pi r}{(2-\alpha) s(c)} .
\end{aligned}
$$

It is straightforward to verify that $\varphi_{S}(S, c)$ is strictly positive and $\varphi_{c}(S, c)$ is strictly negative for all $S \in[\alpha Q /(2-\alpha), Q]$.

Let $S^{*}(c)$ denote the solution to the equation $\varphi(S, c)=0$ with respect to $S$. For $c=0, S^{*}(c)$ is equal to $\alpha Q /(2-\alpha)$. Since $\varphi_{S}(S, c)$ is strictly positive and $\varphi_{c}(S, c)$ is strictly negative for all $S \in[\alpha Q /(2-\alpha), Q]$, it follows that $S^{*}(c)$ is strictly greater than $\alpha Q /(2-\alpha)$ and strictly increasing for all $c>0$. Moreover, there exists a $\bar{c}>0$ such that $S^{*}(\bar{c})=Q$. It then follows that, for all $c \in(0, \bar{c}), S^{*}(c) \in(\alpha Q /(2-\alpha), Q)$ and a $(Q, S, s)$ equilibrium exists. In contrast, for $c \geq \bar{c}, S^{*}(c) \geq Q$ and a $(Q, S, s)$ equilibrium does not exist.

Let $\left(s^{*}(c), S^{*}(c), T_{1}^{*}(c), T_{2}^{*}(c)\right)$ denote the cutoff prices and travelling times in the $(Q, S, s)$ equilibrium associated with the menu cost $c \in(0, \bar{c})$. Above we proved that $S^{*}(c)$ is strictly increasing in $c$. Since $s^{*}(c) \equiv(\alpha Q-r c / b) /(2-\alpha), s^{*}(c)$ is strictly decreasing and $Q-s^{*}(c)$ is strictly increasing in $c$. Since $T_{1}^{*}(c) \equiv \log \left(Q / S^{*}(c)\right) / \pi$ and $T_{2}^{*}(c) \equiv \log \left(S^{*}(c) / s^{*}(c)\right) / \pi, T_{1}^{*}(c)$ is strictly decreasing and $T_{2}^{*}(c)$ is strictly increasing in c. Moreover, since $T_{1}^{*}(c)+T_{2}^{*}(c)$ is strictly increasing, $T_{1}^{*}(c) /\left(T_{1}^{*}(c)+T_{2}^{*}(c)\right)$ is strictly decreasing in $c$.

(ii) Using the fact that $S=s \exp \left(\pi T_{2}\right)$ and $s=(\alpha Q-r c / b) /(2-\alpha)$, we can write the equation $\varphi(S, \pi)=0$ as

$$
\begin{aligned}
\hat{\varphi}\left(T_{2}, \pi\right) & =\left[\frac{1-e^{-(r+\pi) T_{2}}\left(1+(r+\pi) T_{2}\right)}{(r+\pi)^{2} T_{2}}\right]\left[(\alpha Q-r c / b) e^{\pi T_{2}}-\alpha Q\right] \\
& +\left[\frac{1-e^{-(r+\pi) T_{2}}}{r+\pi}\right] \alpha Q+e^{-r T_{2}}\left(\frac{\alpha Q}{r}-\frac{c}{b}\right)-\frac{a Q}{r}=0 .
\end{aligned}
$$


After some algebraic transformations, (B5) becomes

$$
b \alpha Q M\left(T_{2}, \pi\right)=\operatorname{rcN}\left(T_{2}, \pi\right)
$$

where the function $M\left(T_{2}, \pi\right)$ is defined as

$$
\begin{aligned}
M\left(T_{2}, \pi\right)= & -r-\pi(r+\pi) T_{2}+r e^{-(r+\pi) T_{2}} \\
& +\left(\pi(r+\pi) T_{2}-r\right) e^{-r T_{2}}+r e^{\pi T_{2}}
\end{aligned}
$$

and the function $N\left(T_{2}, \pi\right)$ is defined as

$$
N\left(T_{2}, \pi\right)=r e^{\pi T_{2}}+\left(\pi(r+\pi) T_{2}-r\right) e^{-T_{2}}
$$

Let $M_{T_{2}}\left(T_{2}, \pi\right)$ and $N_{T_{2}}\left(T_{2}, \pi\right)$ denote the partial derivatives of $M\left(T_{2}, \pi\right)$ and $N\left(T_{2}, \pi\right)$ with respect to $T_{2}$. Similarly, let $M_{\pi}\left(T_{2}, \pi\right)$ and $N_{\pi}\left(T_{2}, \pi\right)$ denote the partial derivatives of $M\left(T_{2}, \pi\right)$ and $N\left(T_{2}, \pi\right)$ with respect to $\pi$.

Now, let $T_{2}^{*}(\pi)$ denote the solution to (B5) with respect to $T_{2}$. The derivative of $T_{2}^{*}(\pi)$ with respect to the inflation rate $\pi$ is given by

$$
\begin{aligned}
& T_{2}^{* \prime}(\pi)=\frac{M\left(T_{2}^{*}, \pi\right) N_{\pi}\left(T_{2}^{*}, \pi\right)-M_{\pi}\left(T_{2}^{*}, \pi\right) N\left(T_{2}^{*}, \pi\right)}{N\left(T_{2}^{*}, \pi\right) M_{T_{2}}\left(T_{2}^{*}, \pi\right)-N_{T_{2}}\left(T_{2}^{*}, \pi\right) M\left(T_{2}^{*}, \pi\right)} \\
& \quad=\frac{-T_{2}^{*}\left\{2-(r+\pi) T_{2}^{*}-4 e^{-(r+\pi) T_{2}^{*}}+\left[2+(r+\pi) T_{2}^{*}\right] e^{-2(r+\pi) T_{2}^{*}}\right\}}{\pi\left\{1-(r+\pi) T_{2}^{*}+\left[(r+\pi)^{2} T_{2}^{* 2}-2\right] e^{-(r+\pi) T_{2}^{*}}+\left[1+(r+\pi) T_{2}^{*}\right] e^{-2(r+\pi) T_{2}^{*}}\right\}} .
\end{aligned}
$$

It is easy to verify that the above expression is strictly negative.

Next, let $S^{*}(\pi)$ denote the solution to the equation $\varphi(S)=0$ with respect to $S$, which is given by

$$
S^{*}(\pi)=\frac{\alpha Q-r c / b}{2-\alpha} e^{\pi T_{2}^{*}(\pi)} .
$$

The derivative of $S^{*}(\pi)$ with respect to $\pi$ has the same sign as the derivative of $\pi T_{2}^{*}(\pi)$ with respect to $\pi$, which is given by

$$
\begin{aligned}
& T_{2}^{*}(\pi)+\pi T_{2}^{* \prime}(\pi) \\
& \quad=\frac{T_{2}^{*}\left\{e^{-(r+\pi) T_{2}^{*}}\left[2+(r+\pi)^{2} T_{2}^{* 2}\right]-e^{-2(r+\pi) T_{2}^{*}}-1\right\}}{1-(r+\pi) T_{2}^{*}+\left[(r+\pi)^{2} T_{2}^{* 2}-2\right] e^{-(r+\pi) T_{2}^{*}}+\left[1+(r+\pi) T_{2}^{*}\right] e^{-2(r+\pi) T_{2}^{*}}}
\end{aligned}
$$

It is easy to verify that the above expression is strictly positive and, hence, $S^{*}(\pi)$ is strictly increasing in $\pi$. Moreover, $S^{*}(\pi)$ has the following properties: (i) $S^{*}(\pi)=\alpha Q /(2-\alpha)$ 
for $\pi \rightarrow 0$; (ii) $S^{*}(\pi)>\alpha Q /(2-\alpha)$ for all $\pi>0$; (iii) $S^{*}(\pi)>Q$ for $\gamma \rightarrow \infty$. Since $S^{*}(\pi)$ is a continuous and strictly increasing function of $\pi$, the above properties imply that there exists a $\bar{\pi}>0$ such that, for all $\pi \in(0, \bar{\pi}), S^{*}(\pi) \in(\alpha Q /(2-\alpha), Q)$ and, hence, a $(Q, S, s)$ equilibrium exists. In contrast, for $\pi \geq \bar{\pi}, S^{*}(\pi) \geq Q$ and a $(Q, S, s)$ equilibrium does not exist.

Let $\left(s^{*}(\pi), S^{*}(\pi), T_{1}^{*}(\pi), T_{2}^{*}(\pi)\right)$ denote the cutoff prices and travelling times in the $(Q, S, s)$ equilibrium associated with the inflation rate $\pi \in(0, \bar{\pi})$. We have already established that $S^{*}(\pi)$ is strictly increasing and $T_{2}^{*}(\pi)$ is strictly decreasing in $\pi$. Since $s^{*}(\pi) \equiv(\alpha Q-r c / b) /(2-\alpha), s^{*}(\pi)$ is independent of $\pi$ and so is $Q-s^{*}(\pi)$. Since $T_{1}^{*}(\pi) \equiv \log \left(Q / S^{*}(c)\right) / \pi$, it follows that $T_{1}^{*}(\pi)$ is strictly decreasing in $\pi$. Moreover, the ratio $T_{1}^{*}(\pi) /\left(T_{1}^{*}(\pi)+T_{2}^{*}(\pi)\right)$ is strictly decreasing in $\pi$.

\section{Proof of Theorem 3}

Suppose that $V^{*} \in(c, b \alpha Q / r]$ is a solution to the equation

$$
\begin{aligned}
& {\left[\frac{1-e^{-(r+\pi) T\left(V^{*}\right)}\left(1+(r+\pi) T\left(V^{*}\right)\right)}{(r+\pi)^{2}}\right] \frac{2 b(1-\alpha) Q}{T\left(V^{*}\right)}} \\
& +\left[\frac{1-e^{-(r+\pi) T\left(V^{*}\right)}}{r+\pi}\right] b \alpha Q+e^{-r T\left(V^{*}\right)}\left(V^{*}-c\right)-V^{*}=0,
\end{aligned}
$$

where

$$
T\left(V^{*}\right) \equiv \frac{1}{\pi} \log \left(\frac{b(2-\alpha) Q}{r\left(V^{*}-c\right)}\right) .
$$

Let $S^{*}$ be defined as $Q$. Let $s^{*}$ be defined as in equation $(27)$. Let $F^{*}$ be defined as in equation (26) for $s=s^{*}$ and $S=S^{*}$. Moreover, let $T^{*}$ be defined as $\log \left(S^{*} / s^{*}\right) / \pi$ and let $R(p)$ be defined as in $(22)$ for $F=F^{*}$. In order to establish that the tuple $\left(F^{*}, s^{*}, S^{*}, V^{*}\right)$ constitutes an $(S, s)$ equilibrium, we need to verify that it jointly satisfies the optimality conditions (23)-(25) and the stationarity condition (26). In addition, we need to verify that $s^{*} \in(0, Q)$ and that $F^{*}$ is a CDF with support $\left[s^{*}, S^{*}\right]$.

The tuple $\left(F^{*}, s^{*}, S^{*}, V^{*}\right)$ satisfies the stationarity condition (26) by definition. Moreover, notice that $F^{*}$ is a CDF with support $\left[s^{*}, S^{*}\right]$ because $F^{*}\left(s^{*}\right)=0, F^{*}\left(S^{*}\right)=1$ and $F^{* \prime}(p)>0$ for all $p \in\left[s^{*}, S^{*}\right]$.

The optimality condition (23) is satisfied because $R\left(s^{*}\right)=b(2-\alpha) s^{*}$ and $s^{*}=r\left(V^{*}-\right.$ $c) / b(2-\alpha)$ imply $R\left(s^{*}\right)=r\left(V^{*}-c\right)$. Moreover, notice that $s^{*} \in(0, Q)$ because $V^{*}>c$ 
implies $s^{*}>0$ and $V^{*} \leq b \alpha Q / r$ implies $s^{*}<Q$. The optimality condition (24) is satisfied because equation $(\mathrm{C} 1)$ implies

$$
\begin{aligned}
& \int_{0}^{T^{*}} e^{-r t} R\left(Q e^{-\pi t}\right) d x+e^{-r T^{*}}\left(V^{*}-c\right)-V^{*}=0 \\
& V(0)-V^{*}=0
\end{aligned}
$$

Now, we need to verify that the tuple $\left(F^{*}, s^{*}, S^{*}, V^{*}\right)$ satisfies the stationarity condition (25). That is, we need to verify that the seller's value $V(t)$ is non-greater than $V^{*}$ for all $t \in\left[0, T^{*}\right]$. To this aim, notice that $V(t)$ satisfies the differential equation

$$
\begin{aligned}
& r V(t)=\hat{R}(t)+V^{\prime}(t), \\
& \hat{R}(t) \equiv R\left(Q e^{-\pi t}\right)
\end{aligned}
$$

The function $\hat{R}(t)$ is given by

$$
\hat{R}(t)=e^{-\pi t} b Q\left\{(2-\alpha)-2(1-\alpha)\left[1-\frac{\pi t}{\log \left(Q / s^{*}\right)}\right]\right\} .
$$

The derivative of $\hat{R}(t)$ with respect to $t$ is given by

$$
\hat{R}^{\prime}(t)=\pi e^{-\pi t} b Q\left\{2(1-\alpha)\left[1+\frac{1-\pi t}{\log \left(Q / s^{*}\right)}\right]-(2-\alpha)\right\}
$$

The derivative $\hat{R}^{\prime}(t)$ has the same sign as the term in curly brackets in (C5). It is straightforward to verify that this term is strictly increasing in $t$. Hence, $\hat{R}(t)$ is either strictly decreasing in $t$ over the entire interval $\left[0, T^{*}\right]$, or it is first strictly increasing and then strictly decreasing in $t$. Notice that $\hat{R}(t)$ cannot be increasing for all $t \in\left[0, T^{*}\right]$ because $\hat{R}(0)=b \alpha Q \geq r V^{*}$ and $\hat{R}\left(T^{*}\right)=r\left(V^{*}-c\right)<r V^{*}$.

Consider the phase diagram in Figure 8, which describes the differential equation (C3). The black line passing through the origin denotes the locus of points $(\hat{R}, V)$ such that $V=\hat{R} / r$ and, hence, $V^{\prime}=0$. Any point below the black line is such that $V<\hat{R} / r$ and, hence, $V^{\prime}<0$. Any point above the black line is such that $V>\hat{R} / r$ and, hence, $V^{\prime}>0$. From (24), it follows that $\hat{R}(0)=b \alpha Q$ and $V(0)=V^{*} \leq b \alpha Q / r$. Hence, the point $(\hat{R}(0), V(0))$ lies either on or below the black line and $V^{\prime}(0) \leq 0$. From (23), it follows that $\hat{R}\left(T^{*}\right)=r\left(V^{*}-c\right)$ and $V\left(T^{*}\right)=V^{*}-c$. Therefore, the point $\left(\hat{R}\left(T^{*}\right), V\left(T^{*}\right)\right)$ lies on the black line and $V^{\prime}\left(T^{*}\right)=0$.

Now, we want to find out the trajectory that the pair $(\hat{R}(t), V(t))$ follows as it travels 


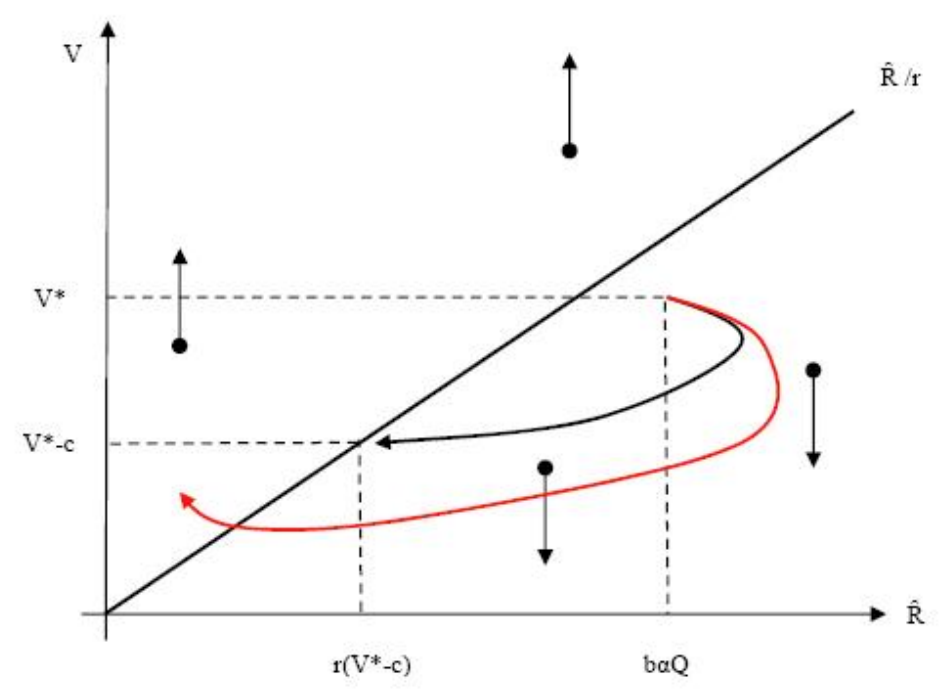

Figure 8: Joint Dynamics of $V(t)$ and $\hat{R}(t)$

from the initial point $\left(b \alpha Q, V^{*}\right)$ to the endpoint $\left(r\left(V^{*}-c\right), V^{*}-c\right)$. First, consider the case $V^{*}<b \alpha Q / r$. In this case, the initial point $\left(b \alpha Q, V^{*}\right)$ lies in the region where $V^{\prime}(t)<0$. For as long as $\hat{R}(t)$ increases, $(\hat{R}(t), V(t))$ moves to the south-east of the initial point $\left(b \alpha Q, V^{*}\right)$. When $\hat{R}(t)$ begins to decrease, $(\hat{R}(t), V(t))$ changes direction and moves towards the south-west and, eventually, it crosses the black line. Suppose that $(\hat{R}(t), V(t))$ crosses the black line at a time $\hat{T}<T^{*}$. Then, after time $\hat{T},(\hat{R}(t), V(t))$ moves to the north-west and, since $\hat{R}(t)$ is decreasing and $V(t)$ is increasing, $(\hat{R}(t), V(t))$ does not reach the black line again. This contradicts the fact that $(\hat{R}(t), V(t))$ reaches the black line at the point $\left(r\left(V^{*}-c\right), V^{*}-c\right)$ at time $T^{*}$. Therefore, $(\hat{R}(t), V(t))$ must first cross the black line at time $T^{*}$ and, hence, $V^{\prime}(t)<0$ for all $t \in\left[0, T^{*}\right]$.

Second, consider the case $V^{*}=b \alpha Q / r$. In this case, it is easy to verify that $(\hat{R}(t), V(t))$ must move first to the south-east, then to the south-west and reach the black line at time $T^{*}$. Also in this case, $V^{\prime}(t) \leq 0$ for all $t \in\left[0, T^{*}\right]$. This completes the proof that the tuple $\left(F^{*}, s^{*}, S^{*}, V^{*}\right)$ is an $(S, s)$ equilibrium. The proof that there is no $(S, s)$ equilibrium if equation $(\mathrm{C} 1)$ does not admit a solution for $V^{*} \in(c, b \alpha Q / r]$ is straightforward and it is omitted for the sake of brevity. 